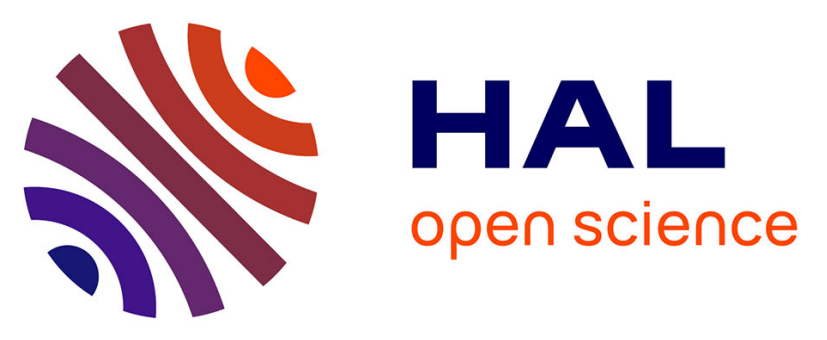

\title{
Target Interneuron Preference in Thalamocortical Pathways Determines the Temporal Structure of Cortical Responses Running title: Target interneuron preference determines thalamocortical dynamics
} y Audrey Hay, Jérémie Naudé, Philippe Fauré, Bertrand Lambolez

\section{- To cite this version:}

y Audrey Hay, Jérémie Naudé, Philippe Fauré, Bertrand Lambolez. Target Interneuron Preference in Thalamocortical Pathways Determines the Temporal Structure of Cortical Responses Running title: Target interneuron preference determines thalamocortical dynamics. Cerebral Cortex, 2019, 29 (7), pp.2815-2831. 10.1093/cercor/bhy148 . hal-02289370

\section{HAL Id: hal-02289370 \\ https: / hal.sorbonne-universite.fr/hal-02289370}

Submitted on 16 Sep 2019

HAL is a multi-disciplinary open access archive for the deposit and dissemination of scientific research documents, whether they are published or not. The documents may come from teaching and research institutions in France or abroad, or from public or private research centers.
L'archive ouverte pluridisciplinaire HAL, est destinée au dépôt et à la diffusion de documents scientifiques de niveau recherche, publiés ou non, émanant des établissements d'enseignement et de recherche français ou étrangers, des laboratoires publics ou privés. 
Title: Target Interneuron Preference in Thalamocortical Pathways Determines the Temporal Structure of Cortical Responses

Running title: Target interneuron preference determines thalamocortical dynamics

Authors: Y. Audrey Hay, Jérémie Naudé, Philippe Faure, Bertrand Lambolez

\begin{abstract}
Affiliation:
Sorbonne Universités, UPMC Univ Paris 06, INSERM, CNRS, Neuroscience Paris Seine Institut de Biologie Paris Seine (NPS - IBPS), 75005 Paris, France

Correspondence to: Audrey Hay (audrey.hay@normalesup.org) or Bertrand Lambolez (bertrand.lambolez@upmc.fr), Université Pierre et Marie Curie, UMR8246, Neuroscience Paris Seine, 9 quai St Bernard case 16, 75005 Paris France. Phone: +33 14427 38 72, Fax: +33 144272584
\end{abstract}

Main text: 44 pages and 7 figures. Number of words: abstract 189, introduction 455, discussion 1359

Supplementary information: supplementary text and 5 supplementary figures

Conflict of Interest: "The authors declare no competing financial interests."

Funding: This work was supported by Centre National de la Recherche Scientifique, Institut National de la Santé et de la Recherche Médicale, Université Pierre et Marie Curie-P6 (Emergence 2012 grant to P.F.) and by grants from Ecole des Neurosciences de Paris ("Network for Viral Transfer"), Fondation pour la Recherche Médicale, Fondation Bettencourt Schueller, Fondation pour la Recherche sur le Cerveau / Rotary Club de France, and Agence Nationale de la Recherche ("IHU Institut de Neurosciences Translationelles de Paris", ANR-10-IAIHU-06). The funders had no role in study design, data collection and analysis, decision to publish, or preparation of the manuscript.

Acknowledgements: We thank Bénédicte Babayan, Bruno Cauli, Bruno Delord, Pascal Legendre, Uwe Maskos, Stéphanie Pons, Martine Soudan, Ludovic Tricoire and the IBPS Imaging Facility for their valuable help. 
ABSTRACT (189 words)

Sensory processing relies on fast detection of changes in environment, as well as integration of contextual cues over time. The mechanisms by which local circuits of the cerebral cortex simultaneously perform these opposite processes remain obscure. Thalamic "specific" nuclei relay sensory information, whereas "non-specific" nuclei convey information on the environmental and behavioral context. We expressed channelrhodopsin in the ventrobasal specific (sensory) or the rhomboid non-specific (contextual) thalamic nuclei. By selectively activating each thalamic pathway, we found that non-specific inputs powerfully activate adapting (slow-responding) interneurons but weakly connect fast-spiking interneurons, whereas specific inputs exhibit opposite interneuron preference. Specific inputs thereby induce rapid feedforward inhibition that limits response duration, whereas, in the same cortical area, non-specific inputs elicit delayed feedforward inhibition that enables lasting recurrent excitation. Using a mean-field model, we confirm that cortical response dynamics depends on the type of interneuron targeted by thalamocortical inputs, and show that efficient recruitment of adapting interneurons prolongs the cortical response and allows the summation of sensory and contextual inputs. Hence, target choice between slow- and fast-responding inhibitory neurons endows cortical networks with a simple computational solution to perform both sensory detection and integration. 


\section{INTRODUCTION (455 words)}

Cortical circuits can respond to incoming signals with high temporal precision, but can also exhibit persistent activity after removal of the stimulus (Goel and Buonomano 2014). The cellular and network properties that enable different inputs to induce brief or long-lasting responses in the same cortical area are still poorly understood. Inhibitory neurons shape the dynamics of cortical responses and are broadly classified as fast-spiking (FS) and adapting (Ad, also termed regular-spiking) interneurons (Kawaguchi 1995, Cauli et al. 1997, Ascoli et al. 2008). Although Ad interneurons are diverse, they collectively exhibit conspicuously slower intrinsic and synaptic activation time constants than FS interneurons (Kawaguchi 1995, Cauli et al. 1997, Geiger et al. 1997, Hu et al. 2010, Goldberg et al. 2011) and are thought to mediate primarily cortico-cortical feedback inhibition (Beierlein et al. 2003, Kapfer et al. 2007).

The thalamus is the major information gateway to the cerebral cortex. It comprises "specific" nuclei that convey primary sensory inputs and "non-specific" nuclei that signal higher order information on the environmental and behavioral context (Van der Werf et al. 2002, Jones 2007, Saalmann 2014, Ito et al. 2015). Specific thalamocortical (TC) inputs excite more strongly FS interneurons than Ad interneurons (Agmon and Connors 1992, Porter et al. 2001, Gabernet et al. 2005, Tan et al. 2008, Ji et al. 2016). The prominent recruitment of FS interneurons by sensory TC inputs causes a rapid feedforward inhibition that results in brief cortical responses (Swadlow 2003, Gabernet et al. 2005, Cruikshank et al. 2007), well suited to detect changes in the sensory flow. The tortuosity of non-specific TC projections has hampered such dissection of their properties in TC slices, but the development of optogenetic tools now allows overcoming this limitation (Zhang et al. 2007, Cruikshank et al. 2012, Hay et al. 2014, Audette et al. 2017). The non-specific rhomboid ( $\mathrm{Rh}$ ) nucleus of the thalamic midline is involved in 
arousal and spatial cognition (Hembrook and Mair 2011, Loureiro et al. 2012, Cholvin et al. 2013, Hallock et al. 2013). We recently reported that direct cortical responses to optogenetic stimulation of Rh axons effectively summate with cortico-cortical excitation (Hay et al. 2014), suggesting that the duration of these TC responses provides a large time-window for integration.

Here, we aim at identifying properties of specific and non-specific TC microcircuits that determine their different cortical response time-course. The projections of the $\mathrm{Rh}$ and those of the ventrobasal (VB) somatosensory relay nucleus converge onto associative area of the parietal cortex (Vertes et al. 2006, Jones 2007). Using optogenetic paradigms in slices of parietal cortex coupled with a computational approach, we show that specific VB and non-specific Rh inputs both robustly connect excitatory neurons, but differentially recruit FS and Ad interneurons with opposite consequences on neuronal network dynamics. 


\section{MATERIALS AND METHODS}

\section{Expression of Channelrhodopsin in neurons of the rhomboid and ventrobasal thalamic nuclei}

All experiments were carried out in accordance with the guidelines published in the European Communities Council Directive of 24 November 1986 (86/609/EEC) and animal protocol has been approved by the local ethics committee (Ce5/2014/00189). Recombinant lentivirus was chosen as an expression vector for these experiments because of its large size/small diffusion (Osten et al. 2006). Expression of channelrhodopsin 2 (ChR2) in thalamic neurons was performed essentially as described (Cruikshank et al. 2012, Hay et al. 2014). Lentivirions encoding a fusion protein containing ChR2 and a yellow variant (YFP) of the green fluorescent protein (GFP) from Aequoria victoria [pLenti-synapsin-hChR2(H134R)-EYFP-WPRE, (Zhang et al. 2007)] were prepared by the platform of the Network for Viral Transfer of the Ecole des Neurosciences de Paris (P24 titer: $50-150 \mathrm{mg} / \mathrm{mL}$ ) as described (Maskos et al. 2005). Male wistar rats (Janvier, 12-13 days old) were deeply anesthetized with an intraperitoneal injection of ketamine and xylasine ( $25 \mathrm{mg}$ and $2.5 \mathrm{mg}$ per $\mathrm{kg}$ body weight respectively). Rats were restrained in a stereotaxic apparatus (David Kopf instruments). The skull was exposed under aseptic conditions and a small burr hole was drilled at coordinates $\mathrm{AP}=-1.8 \mathrm{~mm}$ and $\mathrm{ML}=1.8$ $\mathrm{mm}$ relative to bregma for the rhomboid $(\mathrm{Rh})$ nucleus and $\mathrm{AP}=-1.8 \mathrm{~mm}$ and $\mathrm{ML}=2.6 \mathrm{~mm}$ relative to bregma for the ventrobasal (VB) nucleus. The lentivirion suspension ( 0.8 to $1.5 \mu \mathrm{L})$ was injected with a canula (36G70 (canula) and 26G50 (guide) from Cooper) at a depth of 5.5 $\mathrm{mm}$ and with an angle of $20^{\circ}$ from the medial plane in the Rh nucleus or at a depth of $5.1 \mathrm{~mm}$ without angle in the VB nucleus. The canula was slowly pulled out 5 minutes after the end of the injection and the skull skin was sutured. Animals were allowed to recover with their mother and were housed in a BL-2 facility for at least 3 weeks with free access to food and drink. 


\section{Slice preparation}

Three to four weeks after injection, rats were deeply anaesthetised with an intraperitoneal injection of ketamine and xylasine (100 $\mathrm{mg}$ and $25 \mathrm{mg}$ per $\mathrm{kg}$ body weight respectively) and $120 \mathrm{U}$ of heparine (Sigma) was injected in the left heart to prevent coagulation. Then, rats were intracardiacally perfused with cold $\left(4^{\circ} \mathrm{C}\right)$ sucrose artificial cerebrospinal fluid (ACSF) containing (in mM): sucrose 30, glucose 2.5, $\mathrm{NaCl}$ 126, $2.5 \mathrm{KCl}, \mathrm{NaHPO}_{4}$ 26, $\mathrm{NaH}_{2} \mathrm{PO}_{4} 3$, $\mathrm{MgCL}_{2} 3$ and kynurenic acid 3. Brains were quickly removed and coronal slices (300 $\mu \mathrm{m}$ thick) comprising the parietal association cortex (as defined in Paxinos and Watson, 2007; anteroposterior coordinates: -3.60 to -4.50 relative to Bregma) were prepared in sucrose ACSF using a vibratome (VT1000S, Leica). Slices were transferred in a chamber containing standard ACSF saturated with $95 \% \mathrm{O} 2$ and 5\% CO2. The composition of the standard ACSF was (in mM): $126 \mathrm{NaCl}, 2.5 \mathrm{KCl} \mathrm{NaHPO} 4$ 26, $\mathrm{NaH}_{2} \mathrm{PO}_{4} 3, \mathrm{MgCL}_{2}$ 1, $\mathrm{CaCl}_{2}$ 2, sucrose 10, glucose 10. Slices were allowed to recover for 1 hour at $37^{\circ} \mathrm{C}$ and were then incubated at room temperature $\left(20-25^{\circ} \mathrm{C}\right)$ until recording.

\section{Photostimulation}

ChR2-YFP-expressing neuronal somata and fibers were visualized with a $535 \mathrm{~nm}$ LED (CoolLed) and an YFP filter set (YFP-2427B-000, Semrock). Photoactivation of ChR2 (1-2 ms light pulses) was performed through a GFP filter set (Olympus) using a $465 \mathrm{~nm}$ LED (maximal light power $1.5 \mathrm{~mW} / \mathrm{mm}^{2}$, yielding $2.5 \mathrm{~mW}$ at the focal plane of the $60 \mathrm{x}$ objective) driven by the data-acquisition software pClamp 10.2 through the Digidata interface board (Molecular Devices). Photostimulation was performed at maximal light power for $1 \mathrm{~ms}$ unless otherwise stated. 


\section{Whole-cell recordings}

Slices were transferred to a recording chamber and continuously superfused at $2 \mathrm{ml} / \mathrm{min}$ with oxygenated standard ACSF at room temperature. Patch pipettes (3-6 M $\Omega$ ) were pulled from borosilicate glass (Harvard Apparatus LTD) on a micropipette puller (Model PP-83, Narishige) and filled with $8 \mu \mathrm{l}$ of internal solution containing (in $\mathrm{mM}$ ): $144 \mathrm{~K}$-gluconate, $3 \mathrm{MgCl}_{2}, 10$ HEPES, 0.5 EGTA and $3 \mathrm{mg} / \mathrm{mL}$ biocytin. The $\mathrm{pH}$ was adjusted to 7.2 and the osmolarity to 295 mOsm. For some experiments, we used a Cs-based internal solution containing (in $\mathrm{mM}$ ): 130 Cs-gluconate, $3 \mathrm{MgCl}_{2}$, 10 HEPES, 10 EGTA and $2 \mathrm{mg} / \mathrm{mL}$ biocytin. Recordings began 810 minutes after passing into whole-cell configuration to allow the diffusion of the cesium and the blockade of potassium channels. Whole-cell patch-clamp recordings were made from neurons visualized under infrared videomicroscopy with Nomarski optics. Whole-cell recordings were performed at room temperature $\left(20-25^{\circ} \mathrm{C}\right)$ using a patch-clamp amplifier (Multiclamp 700B, Molecular Devices) connected to a Digidata 1440A interface board (Molecular Devices). Signals were amplified and collected using the data-acquisition software pClamp 10.2. Resting membrane potential was measured just after passing into whole-cell configuration, and only cells with a resting membrane potential more hyperpolarized than -50 $\mathrm{mV}$ were selected. Membrane potentials were not corrected for junction potential. For the test of their firing behavior, cells were maintained at $-60 \mathrm{mV}$ by continuous current injection and submitted to depolarizing current pulses. For the recording of post-synaptic potentials, cells showing a variation of access resistance of more than $20 \%$ were excluded from the study. Recordings of post-synaptic currents were performed at a holding potential of $-60 \mathrm{mV}$ unless otherwise stated. Signals were filtered at $1-5 \mathrm{kHz}$, digitized at $20 \mathrm{kHz}$, saved to a personal computer, and analyzed off-line with Clampfit 10.2 software (Molecular Devices). Responses to single or repetitive photostimulation were quantified with respect to the baseline immediately preceding the photostimulus. Delays-to-onset of EPSCs were measured from the beginning of 
the photostimulus. Results are presented as mean \pm standard error of mean. Between-group comparisons were performed using Mann-Whitney nonparametric or parametric test that does not assume equal standard deviations. A p. value below 0.05 was considered statistically significant.

\section{AMPA/NMDA ratio and decay kinetics}

To determine the AMPA/NMDA ratio of thalamocortical EPSCs, L6 pyramidal neurons were recorded with an intracellular solution containing (in $\mathrm{mM}$ ): 130 CsGluconate, $3 \mathrm{MgCl}_{2}, 10$ HEPES, 10 EGTA and $2 \mathrm{mg} / \mathrm{mL}$ biocytin. The $\mathrm{pH}$ was adjusted to 7.2 and the osmolarity to 295 mOsm. Recordings began 8-10 minutes after passing into whole-cell configuration to allow the diffusion of the intracellular solution and the blockade of potassium channels. Photoinduced EPSCs were recorded at -70 and $+50 \mathrm{mV}$, corresponding to -81 and $+39 \mathrm{mV}$ after correction of the junction potential (11 mV in our conditions) in the presence of GABA-A receptor antagonist (Gabazine, $1 \mu \mathrm{M}$ ). Repetitive photostimulation (150-300 trials, interstimulus interval $1.5 \mathrm{~s}$ ) was performed at each potential. The intensity of the photostimulation was adjusted just below the threshold generating polysynaptic activity and mixed mono/polysynaptic events that sometimes occurred were discarded from the analysis. Only EPSCs occurring within 5ms after the photostimulus were considered. Measurements of AMPAR and NMDAR-mediated currents were based on the assumptions that (i) currents measured at $-70 \mathrm{mV}$ are AMPAR-mediated (due to the voltage-dependent block of NMDAR currents), (ii) the time-to-peak of AMPAR currents is the same at $-70 \mathrm{mV}$ and at $+50 \mathrm{mV}$, and (iii) NMDAR currents can be measured at $+50 \mathrm{mV}$ due to their slower decay with respect to AMPAR currents. Baseline-substracted EPSCs (20 to 150 individual events) were averaged at each potential. The time-to-peak of the AMPA current was measured at $-70 \mathrm{mV}$. The amplitude of the AMPA current at $+50 \mathrm{mV}$ was measured at its extrapolated time-to-peak. The NMDA 
current was measured on a $10 \mathrm{~ms}$ time-window beginning $40 \mathrm{~ms}$ after the photostimulus. Decay slopes of light-evoked currents obtained at $-70 \mathrm{mv}$ and $+50 \mathrm{mV}$ were fitted using a LevenbergMarquardt algorithm with a single exponential $(-70 \mathrm{mV})$ or a linear combination of two exponential functions $(+50 \mathrm{mV})$ of the form $\mathrm{A} \times \exp (-\mathrm{t} / \tau)$, where $\mathrm{A}$ is the amplitude and $\tau$ is the time constant.

\section{Non-stationary noise analysis}

To determine the properties of single channels involved in AMPAR- and NMDAR-mediated thalamocortical EPSCs, non-stationary noise analysis was performed on fully discriminated mono-synaptic events as described (Sigworth 1980; Hartveit and Veruki, 2007). EPSCs were recorded at $-70 \mathrm{mV}$ using KGluconate intracellular solution (AMPAR) or at $+50 \mathrm{mV}$ using CsGluconate intracellular solution (NMDAR). The intensity of the photostimulation (150-300 trials, inter-stimulus interval $1.5 \mathrm{~s}$ ) was adjusted just below the threshold generating polysynaptic activity (corresponding to $1-3 \%$ of maximal light intensity) and mixed mono/polysynaptic events that sometimes occurred were discarded from the analysis. Only EPSCs occurring within 5ms after the photostimulus were considered. Roughly 100 individual EPSCs were selected for the non-stationary noise analysis of each neuron. EPSCs were aligned on the photostimulus and analysis was performed on the decaying phase of EPSCs. Currents were normalized to the mean peak amplitude and the variance over time of the decay phase was determined using the clampex software (Molecular Devices). Baseline noise variance was subtracted. A time-to-time plot of the variance at time $t\left(\sigma^{2}(t)\right)$ as a function of the averaged current amplitude at time $\mathrm{t}\left(\mathrm{I}_{\mathrm{Amp}(\mathrm{t})}\right)$ was fitted by a parabolic curve ( 2 magnitude polynomial function) of the form:

$$
\sigma^{2}(t)=-I^{2} A m p(t) / N+i^{*} I_{A m p}(t)
$$


where $\mathrm{i}$ is the elementary current of the receptor channel, $\mathrm{N}$ is the total number of channels and $\sigma^{2}$ baseline is the variance of the baseline noise. Then, the single channel conductance $(\gamma)$ was calculated as follows:

$$
\gamma=\mathrm{i}^{*}\left(\mathrm{~V}_{\text {hold }}-\mathrm{E}_{\mathrm{Na}}{ }^{+} / \mathrm{K}^{+}\right)
$$

where $V_{\text {hold }}=-80 \mathrm{mV}$ after correction for the junction potential $(11 \mathrm{mV})$ and $\mathrm{E}_{\mathrm{Na}}{ }^{+} / \mathrm{K}^{+}=0 \mathrm{mV}$.

\section{Intracellular labeling and immunohistochemistry}

After electrophysiological recordings, slices were fixed overnight at $4{ }^{\circ} \mathrm{C}$ in $4 \%$ paraformaldehyde/ 0.1 M phosphate buffer $\mathrm{pH} 7.4$ and then rinsed in phosphate buffer saline (PBS). Residual aldehyde was inactivated by incubation with $50 \mathrm{mM} \mathrm{NH} 4 \mathrm{Cl} / \mathrm{PBS}$ solution for $10 \mathrm{~min}$ at room temperature followed by several washes in PBS. Slices were blocked for $2 \mathrm{~h}$ in PBS-GT (gelatine, 2\%; triton, 0.25\%) and then incubated with chicken anti-GFP (1:2000, Aves labs), mouse anti-PV (1:1000, Sigma Aldrich), rabbit anti-NPY (1:1000, a generous gift from Betty Eipper, University of Connecticut Health Center, Farmington, CT; code JH3), mouse anti-SOM (1:1000, Millipore; clone YC7; MAB354) or guinea pig anti-VIP (1:1000, Peninsula Laboratories; T-5030) antibodies for 3 days at $4^{\circ} \mathrm{C}$. After several washes in PBS-GT, slices were incubated overnight at $4^{\circ} \mathrm{C}$ with donkey anti-chicken Alexafluor488 (1:400; Jackson immunoresearch), anti-mouse Alexafluor 647 (1:400; Invitrogen), anti-rabbit Alexafluor 647 (1:400; Invitrogen), anti-mouse Alexafluor 555 (1:400; Invitrogen), anti-guinea pig Alexafluor 647 (1:400; Invitrogen), streptavidin Alexafluor 488 (1:400; Invitrogen) or streptavidin Alexafluor 555 (1:400; Invitrogen) diluted in PBS-GT. Slice were washed in PBS, then incubated 20 min with DAPI (100 ng/mL, Invitrogen) and thereafter extensively washed in PBS. Finally, slices were mounted on gelatin-coated slides in Fluoromount-G (Southernbiotech). Fluorescence images were acquired with a laser scanning confocal microscope (SP5, Leica) using 488, 561 and 633 nm lasers, and analyzed using ImageJ. 


\section{Drugs}

Drugs and chemicals were obtained from Sigma Aldrich except tetrodotoxin (TTX) from Latoxan and orexin B from Tocris. All drugs were applied through the bath perfusion.

\section{Mean field modeling of thalamic inputs onto the cortical network}

In order to assess the computational and functional consequences of the experimental data, we built a neural population model of the parietal cortex using the mean-field approach (Wilson \& Cowan, 1972, Dayan \& Abbott, 2001).

First, we aimed at deciphering the dynamical consequences of the biophysical properties of the different interneurons of the parietal cortex. We considered the simplest possible models for excitatory-inhibitory interactions, with a single inhibitory population, where the instantaneous firing rates of each population at time $\mathrm{t}, \mathrm{R}_{\mathrm{a}}(\mathrm{t})$, is given by:

$\tau_{\mathrm{a}} \frac{\mathrm{dR}_{\mathrm{a}}(\mathrm{t})}{d t}=-\mathrm{R}_{\mathrm{a}}(\mathrm{t})+\left[\mathrm{J}_{\mathrm{a}, \mathrm{e}} \mathrm{R}_{\mathrm{c}}(\mathrm{t})-\mathrm{J}_{\mathrm{a}, \mathrm{i}} \mathrm{R}_{\mathrm{i}}(\mathrm{t})\right]_{+}$

where $\tau_{\mathrm{a}}$ denotes the time constant of firing dynamics of the neurons in population $a=$ (excitatory, inhibitory), henceforth called "firing time constant". The firing time constant reflects how fast the firing rate of one population of neurons, is affected by the firing rate of the other populations. Hence, it depends on passive (membrane time constant) and active (voltagegated conductances) intrinsic properties, as well as synaptic kinetics (Wilson \& Cowan, 1972, Dayan \& Abbott, 2001). The strength $\mathrm{J}_{\mathrm{a}, \mathrm{b}}$ represents the average coupling from neural population $b$ to $a$, and [ $]_{+}$is the static transfer function, that we assumed to be threshold-linear ( $[\boldsymbol{x}]_{+}=\boldsymbol{x}$ if $\boldsymbol{x} \geq 0$, and 0 otherwise) for simplicity. We used standard methods to solve the system analytically for expressing the steady state of network activity, its stability and the eigenvalues $\lambda_{1,2}$ in terms of biophysical parameters of the interneurons population: 
$\lambda_{1,2}=\frac{1}{2}\left(\frac{\left(J_{e e}-1\right)}{\tau_{e}}-\frac{\left(J_{i i}+1\right)}{\tau_{i}}\right) \pm \sqrt{\left(\left(\frac{\left(J_{e e}-1\right)}{\tau_{e}}-\frac{\left(J_{i i}+1\right)}{\tau_{i}}\right)^{2}-\frac{4 F B}{\tau_{e} \tau_{i}}\right)}$

with $F B=J_{i e} J_{a}$ being the strength of the coupling between inhibitory and excitatory populations. For this qualitative investigation, we chose a network with balanced excitation and inhibition $\left(\mathrm{J}_{\oplus \oplus}+\mathrm{J}_{i \theta}=\mathrm{J}_{\Theta i}+\mathrm{J}_{i j}\right)$ and non-trivial recurrent excitation $\left(\mathrm{J}_{\oplus}>1\right.$ and for the figure in the Results, $\mathrm{J}_{\oplus}=\mathrm{J}_{i i}=2$ ). We set $\tau_{\theta}=20$ ms for the rest of this study. Then, equation (2) can be written as a function of the main differing parameters of the interneurons populations, $\boldsymbol{\tau}_{j}$ and $F B$. Hence, we can express the dynamical properties of the model in terms of interneurons' biophysical parameters, as we briefly explain just below [see (Wilson and Cowan 1972) for more information on stability analysis of network models]. Network activity can be stable or not, with and without oscillations, depending on the eigenvalues:

- If one of the eigenvalues is positive, the system is unstable and the network's activity exponentially diverges from its initial condition. In this model, the instability arises from the excitatory feedback onto the excitatory population itself.

- If both eigenvalues are negative, the system is stable and the network's activity converges towards its steady state, which is silent in this model for simplicity.

- If the eigenvalues are complex (i.e. with a non-zero imaginary part) the behavior of the network near its fixed point is oscillatory. In this model, oscillations arise in the case of a stable fixed point, hence the network's activity spirals into the fixed point, corresponding to damped oscillations.

The eigenvalues also describe the speed of exponential convergence (or divergence if the system is unstable) and provide the characteristic time constant of the system, here expressed $\tau_{\mathrm{c}}=\frac{1}{|\mathfrak{R}(\lambda)|}$. The characteristic time constant is of particular importance in this study, as it
as
markedly differs between networks embedding different interneuron populations. The 
imaginary part of the eigenvalue, divided by $2 \pi$, determines the frequency of the oscillations

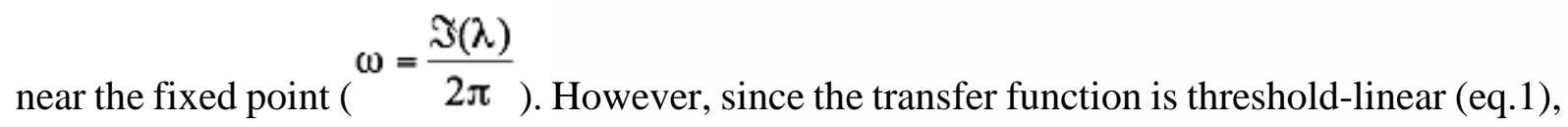
the derivative is discontinuous and the predicted oscillations may not occur. Moreover, the period of the oscillation may be longer than the characteristic time constant of the decay, hence the oscillatory behavior may not be observed, depending on the precise parameters of the model.

We thus investigated the dynamics of a more detailed mean-field model of cortical network, composed of three populations of neurons, one excitatory (pyramidal neurons, $P$ ) and two inhibitory (fast spiking $F$, and adapting $A$ ). The three populations $(i=P, F, A)$ receive average, time-dependent thalamic inputs from the non-specific rhomboid nucleus $(R h), I_{\mathrm{a}, F_{h}}(t)$ and from the specific ventrobasal $(V B)$ nucleus $I_{a, V B}(t)$. These thalamic inputs are excitatory (Fig. 3), hence both $I_{a, F h}(t)$ and $I_{a, V B}(t) \geq 0$ for $a=P, F, A$ and all $t$, but markedly differ in amplitude for the three populations, as we show in this study. The dynamics of thalamo-cortical synapses are included in the time-dependence of the input currents. As we aim at providing a computational comparison of the two types of feedforward inhibition, we did not consider the dynamics induced by corticothalamic feedback. All other types of connections (recurrent and feedback) are potentially present in this model, with strength $J_{a, b}$ representing the average synaptic conductance from neural population $b$ to $a$. In this case, the firing rates of each population at time $t, R_{a}(t)$ are:

$\tau_{\mathrm{a}} \frac{\mathrm{dR}_{\mathrm{a}}(\mathrm{t})}{d t}=-\mathrm{R}_{\mathrm{a}}(\mathrm{t})+\left[\mathrm{I}_{\mathrm{a}, \boldsymbol{F}}(t)+\mathrm{I}_{\mathrm{a}, V B}(t)+\mathrm{J}_{\mathrm{a}, \mathrm{P}} \mathrm{S}_{\mathrm{a}, \mathrm{P}}(\mathrm{t})-\mathrm{J}_{\mathrm{a}, \mathrm{F}} \mathrm{S}_{\mathrm{a}, \mathrm{F}}(\mathrm{t})-\mathrm{J}_{\mathrm{a}, \mathrm{A}} \mathrm{S}_{\mathrm{a}, \mathrm{A}}(\mathrm{t})\right]_{+}$

where $\mathrm{S}_{\mathrm{a}, \mathrm{b}}$ are the average synaptic currents from neural population $b$ to $a$. Synaptic kinetics can play a fundamental role in the dynamical consequences of feedforward inhibition. Hence, 
we separated the synaptic dynamics from the firing time constant. We modeled synaptic currents dynamics by the following equations (Destexhe et al. 1998, Brunel and Wang 2003): $\tau_{X_{a, b}} \frac{\mathrm{dX}_{\mathrm{a}, \mathrm{b}}(\mathrm{t})}{d t}=-\mathrm{X}_{\mathrm{a}, \mathrm{b}}(\mathrm{t})+\mathrm{R}_{b}\left(t-D_{a, b}\right)$ $\tau_{S_{a, b}} \frac{\mathrm{dS}_{\mathrm{a}, \mathrm{b}}(\mathrm{t})}{d t}=-\mathrm{S}_{\mathrm{a}, \mathrm{b}}(\mathrm{t})+\mathrm{X}_{\mathrm{a}, \mathrm{b}}(\mathrm{t})$

where $\boldsymbol{\tau}_{S, b}$ and $\boldsymbol{\tau}_{x_{s, b} \text { are respectively the decay and rise times of average synaptic currents from }}$ population $b$ to $a$, and $D_{a b}$ the delay-to-onset (from presynaptic spike to the onset of the postsynaptic current). Mean-field models of cortical networks usually consider two populations, one excitatory (represented by pyramidal neurons in this study) and one inhibitory. As demonstrated by our experiments, the inhibitory interneurons can be divided in two classes, as they differ by both their responses to thalamic inputs, and by their intrinsic properties. Fastspiking $(F)$ interneurons have a lower firing time constant $\left(\tau_{F}=10 \mathrm{~ms}\right)$ than pyramidal neurons $\left(\tau_{P}=20 \mathrm{~ms}\right.$ ), and receive mostly specific thalamic inputs from the VB. Firing time constant of Adapting $(A)$ interneurons is larger $\tau_{A}=30 \mathrm{~ms}$. Furthermore, the $A$ population receives a larger input from the non-specific thalamus (Rh nucleus). Thalamic inputs $I_{a, F h}(t)_{\text {and }} I_{a, V B}(t)$ were built using the same equations $(4 \mathrm{a}, \mathrm{b})$ as for cortical synaptic currents, with $R_{\mathrm{z}}$ reflecting the activation of the thalamic population $(b=R h, V B)$ during $1 \mathrm{~ms}$. Thalamo-cortical and corticocortical synaptic time constants (onset and decay) and thalamo-cortical coupling strength were fitted from the experimental results obtained in this study. Values for the firing time constants in the detailed model, which depend on a set of intrinsic properties that differ between Ad and FS interneurons, were derived from the literature (Gil and Amitai 1996, Geiger et al. 1997, Gabernet et al. 2005, Huk and Shadlen 2005, Kapfer et al. 2007, Buonomano and Maass 2009, Ibos and Freedman 2014), and we ensured that these parameters give rise to stable dynamics. Parameters for the detailed model were: 
$J_{P, P}=2 ; J_{P, F}=J_{F, P}=1,5 ; J_{P, A}=J_{A, P}=J_{F, F}=J_{F, A}=J_{A, F}=J_{A, A}=J_{P, V B}=J_{P, F \rightarrow}=J_{A, F h}=1 ;$

$J_{A, V B}=0,1 ; J_{F, F,}=0,1 ; \tau_{X_{P, \rho}}=\tau_{X_{F, \rho}}=\tau_{X_{A, \rho}}=\tau_{X_{A, A}}=1 \mathrm{~ms} ;{ }_{X_{P, F}}=\tau_{X_{F, F}}=1,5 \mathrm{~ms} ;{ }_{X_{P, A}}=\tau_{X_{A, \rho}}=2$

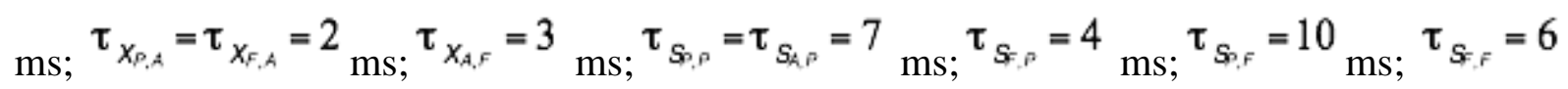

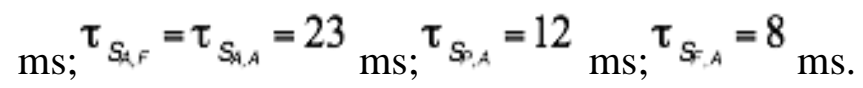

All decay and rise times of thalamocortical synapses were set at $1 \mathrm{~ms}$; except decay times of thalamocortical onto pyramidal cells, set at $4.5 \mathrm{~ms}$. All axonal delays $\mathrm{D}_{\mathrm{a}, \mathrm{b}}$ of corticocortical connections were set at $1 \mathrm{~ms}$. The detailed model was solved numerically using forward Euler method with time step $d t=0.01 \mathrm{~ms}$ in Matlab. 


\section{RESULTS}

\section{Expression of channelrhodopsin in thalamic neurons of the Rh and VB nuclei}

Injection of a lentivirus driving neuronal expression of channelrhodopsin 2 (ChR2) fused to YFP (see Methods) in the Rh or the VB thalamic nuclei resulted in strong labeling of these nuclei (Fig. 1 COLOR and Supplementary Fig. 1), as reported (Cruikshank et al. 2012, Hay et al. 2014). Following injection in the Rh nucleus, YFP fluorescence roughly followed the shape of the Rh nucleus along the vertical, lateral and antero-posterior axes. Fluorescence was nonetheless also observed beyond $\mathrm{Rh}$ borders in the neighbor reuniens midline nucleus, and to a lesser extent in centromedian and submedial nuclei that flank the Rh nucleus (Fig. 1A and Supplementary Fig. 1). Following injection in the VB nucleus, extensive YFP labeling was observed essentially confined within both the ventroposteromedian and ventroposterolateral subnuclei that compose the VB nucleus (Fig. 1B). Transduced neurons exhibited rebound depolarization and action potential bursts typical of thalamic neurons [(Jahnsen and Llinas 1984, Crunelli et al. 1987), $\mathrm{n}=8$ ] and expressed ChR2-YFP at the plasma membrane (Supplementary Fig. 1). Likewise, photostimulation (see Methods) consistently induced bursts of action potentials $(n=8)$ and trains of photostimuli $(10 \mathrm{~Hz})$ elicited repetitive inward currents of sustained amplitude ( $\mathrm{n}=3$, Supplementary Fig. 1). The Rh nucleus is a preferred thalamic target of the wake-promoting neuropeptides orexins (Bayer et al. 2002). In all transduced Rh neurons tested $(n=7)$, application of orexin B $(100 \mathrm{nM})$ converted phasic responses to repetitive photostimulation into a tonic action potential discharge (Supplementary Fig. 1), consistent with the effects of orexin B on the excitability of Rh neurons (Bayer et al. 2002). Hence, a large proportion of Rh and VB neurons expressed functional ChR2 without conspicuous alteration of their membrane properties, as reported (Cruikshank et al. 2012, Hay et al. 2014). 
Throughout the rest of this study, recordings were performed in the parietal association cortex, which receives Rh TC fiber innervation (Fig. 1C) with larger vertical extent than other cortical areas (Vertes et al. 2006) and is also a target of VB TC fibers. Conversely, the parietal association cortex does not receive projections from the centromedian and reuniens neighbor nuclei (Berendse and Groenewegen 1991, Vertes et al. 2006). The mean density of TC fibers was estimated by measuring cortical anti-GFP immunofluorescence intensity in the parietal association cortex (-3.60 to -4.50 relative to Bregma, Paxinos and Watson, 2007) following expression of ChR2-YFP in the Rh or the VB nucleus ( $n=5$ slices from 4 animals, $n=6$ slices from 4 animals, respectively). To ensure localization of cortical layers, anti-GFP immunofluorescence was combined with DAPI staining, whose mean fluorescence profile peaked in layer 4 (L4, $n=5$ slices, Fig. 1D). Immunofluorescence of Rh fibers was maximal in L6, smoothly decreased to reach minimal value in L2/3 and showed a sharp peak in L1 ( $\mathrm{n}=5$ slices, Fig. 1D). This result is consistent with the known distribution of Rh TC fibers across layers in this cortical area (Vertes et al. 2006). Conversely, the mean density of VB TC fibers in the parietal association cortex was maximal in L4 and exhibited a smaller peak in L6 ( $\mathrm{n}=6$, Fig. 1D), in agreement with earlier observations in the somatosensory barrel cortex (Meyer et al. 2010, Wimmer et al. 2010).

\section{Rh TC inputs elicit longer responses of the cortical network than VB TC inputs}

Because VB and Rh nuclei are thought to convey information of different nature to the cortex, we first compared the kinetics of the cortical network response to a single photostimulation (1$2 \mathrm{~ms})$ at maximal light intensity $(5 \mathrm{~mW})$ of either VB or Rh fibers. Recordings were performed in pyramidal neurons from L6 (Fig. 2A), which receives a peak density of both types of TC fibers. The nature and localization of all recorded neurons throughout this study was assessed by their electrophysiological properties and morphology examined by infrared live microscopy 
(Fig. 2A and B) and confirmed by post-hoc revelation of the biocytin contained in the intracellular fluid (Fig. 2A). Photostimulation of thalamic fibers induced a complex excitatory current, reflecting both monosynaptic and polysynaptic network activity, which was abolished by glutamatergic receptor antagonists CNQX and AP5 (n=9, Fig. 2B). We estimated network activation by quantifying the duration of light-induced excitatory and inhibitory post-synaptic currents (EPSCs and IPSCs) at $33 \%$ of maximal amplitude in L6 (Fig. 2 COLOR). EPSCs and IPSCs were isolated from each other by recording at the reversal potential of GABAA $(-70 \mathrm{mV})$ and glutamatergic $(0 \mathrm{mV})$ currents, respectively. We found that $33 \%$ widths of Rh TC EPSCs and IPSCs $(51.5 \pm 13.2 \mathrm{~ms}$ and $131.2 \pm 17.9 \mathrm{~ms}$, respectively; $\mathrm{n}=15)$ were about twice those measured upon VB TC stimulation (26.5 $\pm 2 \mathrm{~ms}$ and $61.4 \pm 8.4 \mathrm{~ms}, \mathrm{n}=14, \mathrm{p}<0.05$; Fig. $2 \mathrm{C}-\mathrm{D})$. These differences were assessed by calculating the ratio of the area vs. the peak amplitude of PSCs, which was significantly larger for Rh than for VB TC EPSCs and IPSCs ( $\mathrm{p}<0.05$ for EPSCs, p<0.01 for IPSCs; Fig. 2C-D). These results indicate that Rh inputs induce longerlasting recurrent activity of the cortical network in L6 than VB inputs. Time-to-peak of induced IPSCs was also markedly longer for Rh TC than for VB TC responses ( $p<0.01$, Fig. $2 C-D)$. This might reflect the summation of inhibitory events or could suggest a differential recruitment of inhibitory interneurons by the two TC inputs. In contrast, no significant difference in EPSC time-to peak was observed ( $\mathrm{p}=0.26)$, which is likely due to the fast kinetics of AMPA currents that do not allow for the efficient summation of events. In the following sections, we aimed at identifying the mechanisms underlying the different time-course of cortical network responses elicited by Rh and VB inputs.

\section{Properties of Rh TC synaptic currents}

The kinetics and voltage-dependence of synaptic currents and the properties of underlying receptor-channels potentially influence the duration of network responses. We examined these 
properties of Rh TC synapses on L6 pyramidal neurons. Although some EPSC traces exhibited an essentially monophasic decay, most clearly showed the occurrence of polysynaptic activity (see examples in Fig. 2 and 3), which impairs the measurement of Rh TC synaptic properties. To isolate direct EPSCs from recurrent cortico-cortical activity without interfering with synaptic properties, we reduced photostimulus intensity to get as close as possible to minimal stimulation condition and obtain monosynaptic events with little contamination from polysynaptic activity. At maximal intensity of stimulation, the mean delay-to-onset values $(\mathrm{n}=41)$ showed a main peak centered around $3 \mathrm{~ms}$, presumably corresponding to direct monosynaptic EPSCs, and responses with longer delays-to-onset consistent with indirect polysynaptic responses (Fig. 3A). When stimulus intensity was gradually declined, the delayto-onset smoothly increased to reach $7.6 \pm 1 \mathrm{~ms}$ at $1 \%$ of maximal stimulation intensity $(\mathrm{n}=6$, Fig. 3B). A roughly parallel increase of EPSC failure rate occurred to reach $25 \pm 9 \%$ at $1 \%$ of maximal light intensity, consistent with a decrease in the effectiveness of $\mathrm{Rh}$ fiber photostimulation. The EPSCs obtained at weak stimulus intensities (1-3\%) exhibited essentially monotonous decays, indicating they were direct monosynaptic events with little contamination from recurrent polysynaptic activity (Fig. 3E). The delay-to-onset of pooled EPSCs obtained at weak stimulus intensity was $5.3 \pm 0.45 \mathrm{~ms}$ ( $\mathrm{n}=6$ neurons). We thus used a $5 \mathrm{~ms}$ window after weak stimulation to select direct EPSCs whereas more delayed events were considered polysynaptic.

We then measured the kinetics of Rh TC synaptic currents in L6 pyramidal cells and the contribution of glutamate receptor-channel subtypes using weak intensity stimulation (1-3\% of maximal light intensity, Fig. 3C-E B\&W). EPSCs were recorded at $-70 \mathrm{mV}$ (i.e. $-81 \mathrm{mV}$ after correction of the junction potential), where the contribution of NMDA receptor (NMDAR)mediated currents is minimal, and at $+50 \mathrm{mV}$ (i.e. $+39 \mathrm{mV}$ ), where both AMPAR and NMDAR contribute to synaptic currents. Indeed, EPSCs recorded at $-70 \mathrm{mV}$ exhibited rapid decays, 
consistent with the fast kinetics of AMPARs, and were abolished by the AMPAR antagonist CNQX $(10 \mu \mathrm{M}, \mathrm{n}=4$; Fig. $3 \mathrm{C})$. Conversely, currents recorded at $+50 \mathrm{mV}$ additionally comprised a slow phase mediated by NMDARs, which was abolished by the NMDAR antagonist AP-V $(50 \mu \mathrm{M}, \mathrm{n}=4$; Fig. $3 \mathrm{C})$. In the presence of AP-V, mean EPSC amplitudes obtained at $-70 \mathrm{mV}$ $(-30.5 \pm 7.7 \mathrm{pA})$ and $+50 \mathrm{mV}(18.2 \pm 4.7 \mathrm{pA}, \mathrm{n}=4)$ were consistent with a linear $\mathrm{I} / \mathrm{V}$ curve indicative of the involvement of calcium-impermeable AMPARs. An index of the NMDAR/AMPAR current ratio at $+50 \mathrm{mV}$ was determined (see Methods), based on the observation that the mean time-to-peak of AMPAR-mediated currents exhibited similar values at $-70 \mathrm{mV}$ and $+50 \mathrm{mV}$ in the presence of $\mathrm{AP}-\mathrm{V}(5.3 \mathrm{~ms}$ and $5.6 \mathrm{~ms}$ respectively, $\mathrm{n}=3)$. The extrapolated AMPAR-mediated current was $22.2 \pm 6.4 \mathrm{pA}$ and the NMDAR-mediated current measured on a $10 \mathrm{~ms}$ time-window beginning $40 \mathrm{~ms}$ after the photostimulus was $13.1 \pm 3.9 \mathrm{pA}$, yielding an NMDA/AMPA ratio of $0.77 \pm 0.06$ ( $n=11$, Fig. 3C). The decays of EPSCs were fitted with a single or a dual exponential (at -70 or $+50 \mathrm{mv}$, respectively; see Methods), yielding $\tau$-AMPA $=7.6 \pm 0.6 \mathrm{~ms}(\mathrm{n}=24)$ and $\tau$-NMDA $=84.2 \pm 12.4 \mathrm{~ms} \quad(\mathrm{n}=12)$. These synaptic properties are highly similar to those reported for VB TC synapses on pyramidal neurons (Gil and Amitai 1996, Gil et al. 1999, Bannister et al. 2005).

Non-stationary noise analysis [(Sigworth 1980), see Methods] was used to determine the properties of unitary AMPAR and NMDAR channels involved at the Rh TC synapse onto L6 pyramidal cells. This analysis was performed on responses obtained at - $70 \mathrm{mV}$ (AMPAR) or $+50 \mathrm{mV}$ (NMDAR) as exemplified in Fig. 4B-C. For the neuron clamped at - $70 \mathrm{mV}$, EPSC amplitudes ranged between $13 \mathrm{pA}$ and $58 \mathrm{pA}$ (mean amplitude $=28.5 \mathrm{pA}$, Fig. 3D). The mean variance of the baseline noise $\left(1.51 \mathrm{pA}^{2}\right.$, Fig. 3D) was subtracted from the variance of EPSC currents. The plot of the variance versus the mean amplitude was fitted with a parabolic equation, from which we calculated the value of AMPAR single channel conductance. Mean value of AMPAR single channel conductance was $11.5 \pm 1.8 \mathrm{pS}(\mathrm{n}=18)$, consistent with earlier 
observations at the VB TC synapse (Bannister et al. 2005). For EPSCs recorded at $+50 \mathrm{mV}$, similar analysis as above yielded values of NMDAR single channel conductance $(40.5 \pm 2.9$ $\mathrm{pS}, \mathrm{n}=18$, Fig. 3D) that are in the range of those reported for somatodendritic NMDA receptors in pyramidal cells (Binshtock et al. 2006). The absence of NMDA current at -70 $\mathrm{mV}$ and the high mean unitary conductance of NMDARs rule out a substantial contribution of GluN2C/Dcontaining receptors (Binshtock et al. 2006, Hildebrand et al. 2014) that may explain the longlasting cortical responses to $\mathrm{Rh}$ inputs. Hence, the above observations indicate that $\mathrm{Rh} \mathrm{TC}$ synapses are not endowed with peculiar properties that could account for the different duration of L6 cortical responses elicited by Rh and VB TC inputs.

\section{$R \boldsymbol{R} T C$ inputs preferentially target infragranular cortical layers}

The complex pattern of intra- and interlaminar cortico-cortical connections potentially influences the decay of cortical responses to afferent inputs (Thomson and Bannister 2003). We thus asked whether the distribution of Rh TC inputs onto diverse neuronal types across cortical layers could explain the persistence of cortical responses to Rh fiber photostimulation. In order to compare direct $\mathrm{Rh} \mathrm{TC}$ inputs to different cortical layers, we recorded 3-5 cells in a vertical row of each slice (Fig. 4 -COLOR).

To obtain a reliable quantification of direct input amplitude across neurons and slices, light intensity and flash duration had to be set, preventing the use of weak stimulation to isolate direct inputs from recurrent network activation. We thus recorded responses to photostimulation (maximal intensity for $1 \mathrm{~ms}$ ) in the combined presence of TTX and 4-AP $(1 \mu \mathrm{M}$ and $1 \mathrm{mM}$, respectively), which allows for triggering presynaptic glutamate release by $\mathrm{ChR} 2$ while blocking network activity (Hull et al. 2009, Petreanu et al. 2009). Application of TTX alone abolished light-induced responses, which were restored by additional application of 4AP (see examples in Fig. 4A-B). Responses in the presence of TTX-4AP were monophasic and had 
roughly the same amplitude as the direct EPSCs recorded in control condition, but slower onset and decay kinetics (n=6, Fig. 4A-B). Recorded cells were identified as excitatory or inhibitory based on their somatodendritic morphology and dendritic spine density [(Peters and Jones 1984), Fig. 4C and Supplementary Fig. 2]. Excitatory cells were stricto sensu pyramidal neurons in L2/3, L5 and L6 (Peters and Jones 1984), spiny stellate and star pyramidal neurons in L4 (Feldmeyer et al. 1999, Staiger et al. 2004), according to the layer-specific predominance of these morphological subtypes. Inhibitory neurons were characterized by their non-pyramidal morphology, the low density of their dendritic spines and their electrophysiological properties. Subsequently, they were sub-classified as FS or Ad interneurons based on their electrophysiological properties and on the presence (FS) or absence (Ad) of parvalbumin [(Kawaguchi 1995, Cauli et al. 1997), Fig. 4C and Supplementary Fig. 3]. The responsiveness of excitatory neurons roughly followed the profile of Rh TC fiber density with significant differences between layers (Fig. 4D; e.g. L6 > each other layer, L4 < L5 and L6, p<0.05). Responses were minimal in L2/3 and L4 $(24.2 \pm 10.3 \mathrm{pA}$ and $13.3 \pm 3.9 \mathrm{pA}, \mathrm{n}=12$ and 13 , respectively), which contained substantial proportions of unresponsive excitatory neurons (25 and $31 \%$, respectively). Responsiveness increased in L5 (57.9 $\pm 19.3 \mathrm{pA}, 13 \%$ unresponsive cells, $\mathrm{n}=15)$ and reached a maximum in L6 (185.1 $\pm 39.4 \mathrm{pA}, 5 \%$ unresponsive cells, $\mathrm{n}=21)$. In L1, which contains only GABAergic interneurons, all cells responded to photostimulation, consistent with the peak of $\mathrm{Rh}$ fiber density in this layer, but with moderate intensity $(35.7 \pm$ 7.9 pA, n=10). Responsiveness of interneurons (Ad and FS pooled, Fig. 4D) across layers followed the same pattern as that of excitatory neurons. L2/3 interneurons were weakly activated by $\mathrm{Rh}$ TC inputs $(7.3 \pm 2.2 \mathrm{pA}, 33 \%$ unresponsive cells, $\mathrm{n}=12)$, whereas L5 and L6 interneurons showed strong responses and high response probability (L5 interneurons: $89.0 \pm$ 41.7 pA, $5.3 \%$ unresponsive cells, n=19; L6 interneurons: $142.4 \pm 39.8$ pA, $10.3 \%$ unresponsive cells, $\mathrm{n}=29$ ). Hence, $\mathrm{Rh}$ TC inputs strongly target infragranular layers but 
virtually avoid L4, similar to other non-specific nuclei but in marked contrast with specific inputs, which prominently connect L4 in addition to infragranular layers (Herkenham 1980, Wimmer et al. 2010, Meyer et al. 2010, Constantinople and Bruno 2013). The weakness of Rh TC inputs on neurons of L2-4 suggests that these layers do not significantly contribute, for instance through feedforward excitation, to the persistence of cortical network responses we observed in L6.

\section{Rh and VB TC inputs exhibit opposite preference for Ad and FS interneurons}

The above observations indicate that the different time-course of L6 responses to Rh and VB TC inputs relies on the differential activation of infragranular cortical circuits by these inputs. We thus compared the responses of infragranular neurons to Rh and VB TC inputs (Fig. 5 $\mathrm{B} \& \mathrm{~W})$. Photostimulation of $\mathrm{Rh} \mathrm{TC}$ fibers in the presence of TTX-4AP elicited EPSCs in a majority of FS and Ad interneurons (87\% of FS and 94\% of Ad neurons, $n=15$ and n=33, respectively), but of markedly smaller amplitude in FS interneurons (Fig. 5A1). Indeed, the response amplitude of Ad interneurons $(159 \pm 40 \mathrm{pA})$ was comparable to that of pooled infragranular pyramidal neurons $(132 \pm 26 \mathrm{pA}, \mathrm{n}=36)$, whereas responses of FS interneurons were significantly smaller $(37 \pm 11 \mathrm{pA}, \mathrm{p}<0.05$, Fig. $5 \mathrm{~A} 1)$. This is in marked contrast with the situation reported for VB TC inputs, which powerfully connect FS interneurons in the somatosensory barrel cortex (Gabernet et al. 2005, Cruikshank et al. 2007). Accordingly, we found in the parietal association cortex that EPSCs induced by photostimulation of VB TC fibers in the presence of TTX-4AP were larger in FS neurons $(143.3 \pm 23.9 \mathrm{pA}, \mathrm{n}=6)$ than in pyramidal neurons $(98.6 \pm 40.4 \mathrm{pA}, \mathrm{n}=19)$, and had smallest amplitude in Ad neurons $(20.2 \pm$ $6.5 \mathrm{pA}, \mathrm{n}=24$, Fig. 5A2), in line with previous observations using optogenetic or electrical stimulation of VB inputs to the barrel cortex (Beierlein et al. 2003, Gabernet et al. 2005, Cruikshank et al. 2007, Cruikshank et al. 2010). These results indicate that Rh inputs 
preferentially target Ad over FS interneurons and confirm that VB inputs exhibit opposite preference.

The population of L6 Ad interneurons comprises two major classes: cells expressing both somatostatin and neuropeptide Y (SOM interneurons) and cells expressing neuropeptide Y but not somatostatin (NPY interneurons); as well as a minor class of cells expressing vasoactive intestinal peptide (VIP interneurons), which are devoid of SOM and NPY and represent less than $5 \%$ of all L6 interneurons (Perrenoud et al. 2013). In order to identify the Ad interneuron subtypes targeted by thalamic projections, we probed immunoreactivity for these neuropeptides in L6 interneurons tested for responsiveness to photostimulation of either Rh or VB TC fibers in the presence of TTX-4AP (Fig. 5B and Supplementary Fig. 2B). Putative FS neurons, identified from their electrophysiological properties (see Supplementary results and Supplementary Fig. 3), were discarded from this analysis. NPY was detected in 17 out of 32 neurons analyzed (52\%, Fig. 5B2 and Supplementary Fig. 2B), among which 11 and 6 were tested for Rh and VB input responsiveness, respectively. SOM was detected in 17 out of 131 neurons analyzed (13\%, Fig. 5B1 and Supplementary Fig. 2B), comprising 12 Rh-tested and 5 VB-tested neurons. Both NPY+ and SOM+ Ad interneurons received Rh inputs (EPSC amplitude: $160.1 \pm 41.5 \mathrm{pA}$ for NPY+ cells and $106.9 \pm 52.0 \mathrm{pA}$ for SOM+ cells, Fig. 5B3), but were only weakly connected by VB inputs (EPSC amplitude: $23.3 \pm 13.3$ pA for 3 responsive out of $6 \mathrm{NPY}+$ cells, and $40 \mathrm{pA}$ for 1 responsive out of $4 \mathrm{SOM}+$ cells). As exemplified in Supplementary Fig. 2B, the somatodendritic morphologies of these interneurons corresponded to those reported for SOM (bipolar/bitufted or multipolar with thick primary dendrites) and NPY (neurogliaform-like with thin and ramified dendrites) interneuron types in L6 (Perrenoud et al. 2013). SOM+ cells ( $n=17)$ were either bipolar/bitufted or multipolar $(n=9$ and 8 , respectively). A majority of NPY+ cells were also bipolar/bitufted or multipolar $(\mathrm{n}=7$ and 7 out of 17, respectively) consistent with NPY expression in SOM interneuron type 
(Perrenoud et al. 2013), but 3 cells exhibited neurogliaform-like morphology typical of the NPY interneuron type (Supplementary Fig. 2B). Finally, we examined VIP expression in Ad interneurons responsive to $\mathrm{Rh}$ stimulation. None of the 63 interneurons tested exhibited detectable immunoreactivity for this neuropeptide. These results indicate that $\mathrm{Rh}$ inputs to $\mathrm{L} 6$ target both NPY and SOM interneurons, which are the major Ad interneuron subtypes in this layer (Perrenoud et al. 2013). Conversely, these Ad interneuron types are only weakly connected by VB inputs to L6.

\section{Rh and VB TC inputs elicit different temporal sequences of excitation/inhibition}

We next compared the recruitment of L6 FS and Ad interneurons by Rh and VB TC inputs using current-clamp recordings (Fig. 6A COLOR). To avoid biases caused by resting potential variability, membrane potential was set at $-60 \mathrm{mV}$ using continuous current injection (Resting potentials: VmAd-Rh=-63.3 $\pm 1.0 \mathrm{mV}$ and $\mathrm{VmAd}-\mathrm{VB}=-59.9 \pm 0.5 \mathrm{mV}, \mathrm{n}=11$ and 6 , respectively, $\mathrm{p}=0.57 ; \mathrm{VmFS}-\mathrm{Rh}=-59.4 \pm 0.5 \mathrm{mV}$ and $\mathrm{VmFS}-\mathrm{VB}=-59.3 \pm 1.4 \mathrm{mV}, \mathrm{n}=9$ and 11 , respectively, $\mathrm{p}=0.96$ ). Action potentials followed photostimulation in all FS neurons tested, but with a much shorter delay for VB than for Rh inputs (delay-to-spike $=4.1 \pm 0.5 \mathrm{~ms}$ and 10.6 $\pm 1.5 \mathrm{~ms}, \mathrm{n}=6$ and 11 , respectively, $\mathrm{p}<0.05$, Fig. $6 \mathrm{~A}$ ). This is consistent with a direct excitation of FS neurons by VB inputs and an indirect excitation of these neurons by Rh inputs. Conversely, spikes occurred in a majority of Ad neurons (6 out of 9, Fig. 6A) upon stimulation of Rh fibers, but in only 1 out of 11 Ad neurons tested upon stimulation of VB fibers (not shown). We further noticed that spikes elicited by $\mathrm{Rh}$ inputs in Ad neurons occurred with a longer delay $(8.9 \pm 1.5 \mathrm{~ms}, \mathrm{n}=6)$ than those triggered by VB inputs in FS neurons $(4.1 \pm 0.5 \mathrm{~ms}$, $n=6, p<0.05$, Fig. 6A), consistent with the different onset slopes of EPSCs and spikes in these interneuron types (Supplementary Fig. 4). These results indicate that Rh and VB TC inputs differentially recruit FS and Ad interneurons. 
We explored the consequences of this differential interneuron targeting on the inhibition of L6 pyramidal neurons (Fig. 6B). The onset of IPSCs was markedly delayed upon Rh fiber stimulation as compared to VB fiber stimulation $(8.4 \pm 1.0 \mathrm{~ms}$ and $4.5 \pm 0.1 \mathrm{~ms}$, respectively; $\mathrm{p}<0.05)$ in spite of comparable amplitudes $(414 \pm 116 \mathrm{pA}, \mathrm{n}=16$ and $629 \pm 139 \mathrm{pA}, \mathrm{n}=15$, respectively; $\mathrm{p}=0.2$ ), consistent with results shown in Fig. 2B. Conversely, EPSCs elicited by Rh and VB inputs did not differ significantly (delays to onset: $2.9 \pm 0.2 \mathrm{~ms}$ and $2.6 \pm 0.2 \mathrm{~ms}$, amplitudes: $287 \pm 85 \mathrm{pA}$ and $269 \pm 61 \mathrm{pA}, \mathrm{n}=16$ and $\mathrm{n}=15$, respectively; $\mathrm{p}>0.2$ for both). These results indicate that the differential recruitment of FS and Ad neurons by VB and Rh inputs results in fast and delayed feedforward inhibition, respectively.

Finally, we analyzed the consequences of the differential interneuron targeting by VB and Rh inputs on L6 network dynamics by plotting the post-stimulus time distribution of excitatory and inhibitory events recorded in this study (Fig. 6C). Upon VB stimulation, pyramidal cell EPSCs, FS neuron spiking and pyramidal cell IPSCs were arranged sequentially in a narrow time window. These results are consistent with VB inputs triggering rapid and synchronous firing of FS neurons, leading to fast feedforward inhibition that temporally limits network excitation, as described (Swadlow 2003, Gabernet et al 2005). Upon Rh TC stimulation, this well-arranged sequence was not observed and events occurred in a far broader time-window (Fig. 6C). The largely overlapping distributions of EPSCs and IPSCs in pyramidal cells, and of these IPSCs and interneuron spiking, suggest that IPSCs resulted from both feedforward recruitment of Ad neurons and feedback excitation of FS cells. These results indicate that the different weights of VB and Rh inputs onto FS and Ad interneurons result in different inhibition kinetics with contrasting results on cortical dynamics.

\section{Computational consequences of the differential activation of FS and Ad interneurons}


We assessed the causal role of target interneuron preference in network response using a meanfield model of the parietal association cortex (Wilson and Cowan 1972, Dayan and Abbott 2001, Fig. 7 COLOR). We first illustrate how network activity depends on interneuron properties (firing time constants, see Methods, and strength of inhibitory feedback) using the analytical solution of a reduced model comprising an excitatory population and an interneuron population. In the following, we only consider regions of the parameters space resulting in stable dynamics. As FS cells have a faster firing time constant (in the reduced model, the firing time constant depends on both synaptic kinetics and intrinsic properties) than Ad interneurons, and a stronger coupling with pyramidal cells (Kawaguchi 1995, Cauli et al. 1997, Hu et al. 2010, Goldberg et al. 2011), pyramidal-FS or pyramidal-Ad couples markedly differed in their characteristic relaxation time-constants to equilibrium (Fig. 7A and Supplementary Fig. 5). Following thalamic stimulation, the pyramidal-FS network quickly relaxes to its basal activity, whereas pyramidal-Ad network slowly decays to its initial state due to recurrent activity. The decay can be associated with damping oscillations, whose frequency depends on the biophysical properties of interneurons (Supplementary Fig. 5).

We next simulated a more detailed mean-field model comprising the three populations (pyramidal, FS and Ad), and additionally separating synaptic kinetics (modeled as separate variables) from intrinsic properties (time constant per se, see Methods). In this model, Rh and VB inputs equally target pyramidal cells, but preferentially activate Ad (Rh) or FS (VB) interneurons, according to experimental data. Upon VB stimulation the model rapidly relaxes to its initial state, while $\mathrm{Rh}$ stimulation evokes a long-lasting network activity with an oscillatory component (Fig. 7B and Supplementary Fig. 5). VB and Rh stimulation yield similar values of pyramidal cell activity at $10 \mathrm{~ms}$ post-stimulus (Fig. 7B), confirming that delayed feedforward inhibition through Ad cells targeting is responsible for the prolonged network response. To assess the role of Ad neurons on prolonged network response, we varied TC input 
weight on Ad cells while TC connection onto pyramidal neurons remained constant. When the weight on Ad cells is set to zero (Pyr-only: connection onto pyramidal cells only), the network response to TC stimulation is brief, due to the feedback from FS neurons (Fig. 7C central panel), but nonetheless longer than the response to VB inputs, confirming the role of feedforward activation of FS interneurons in temporal precision. Furthermore, increasing the weight of TC inputs on Ad interneurons proportionally increases the network response duration (Fig. 7C right panel). Hence, the recruitment of Ad interneurons is crucial for the persistence of responses to Rh inputs because Ad neurons inhibit FS neurons, thereby dampening feedback inhibition of Pyr neurons. Finally, prior activation of Rh inputs increases the peak response to subsequent VB inputs (Fig. 7D), during a time-window that is longer upon recruitment of both Ad and Pyr neurons by Rh inputs than upon Pyr-only activation (Fig. 7D). This suggests that feedforward excitation of Ad interneurons by contextual $\mathrm{Rh}$ inputs enables these inputs to influence the processing of sensory VB inputs by the cortical network. 
DISCUSSION (1359 words)

Our findings reveal an unsuspected role of interneuron diversity and of thalamic targetpreference in the variety of cortical dynamics underlying sensory processing. The distinctive intrinsic and synaptic activation time-constants of FS and Ad interneurons are well described (Kawaguchi 1995, Cauli et al. 1997, Geiger et al. 1997, Hu et al. 2010, Goldberg et al. 2011), but the consequences of their differential recruitment on the time course of cortical responses have been overlooked. Earlier studies established the critical role of FS interneuron-mediated fast feedforward inhibition in limiting the duration of cortical responses to specific thalamic inputs (Swadlow 2003, Gabernet et al. 2005). Here, we show that Ad interneurons do not merely serve in cortico-cortical inhibition, but are also major targets of Rh inputs that mediate delayed feedforward inhibition and enable prolonged cortical responses.

\section{Rh projections robustly activate infragranular layers of the parietal association cortex}

The early view of non-specific thalamic nuclei as a diffuse projection system that globally upregulates cortical activity (Moruzzi and Magoun 1949, Jasper et al. 1953) has been challenged by anatomical characterization of their well-defined and complementary innervation of cortical areas (Berendse and Groenewegen 1991, Vertes et al. 2006, Van der Werf et al. 2002). The cortical lamination of $\mathrm{Rh}$ fibers follows the characteristic pattern of non-specific $\mathrm{TC}$ projections, which markedly differ from sensory TC inputs that strongly target L4 (Vertes et al. 2006, Van der Werf et al. 2002, Herkenham 1980, Wimmer et al. 2010, Meyer et al. 2010, Constantinople and Bruno 2013). Likewise, we found that responses to $\mathrm{Rh} \mathrm{TC}$ inputs were minimal in L4, suggesting that non-specific thalamic nuclei, which convey contextual information (Van der Werf et al. 2002, Saalmann 2014, Ito et al. 2015), may generally have 
little influence on the processing of primary sensory information in this layer, which exhibits the lowest level of context-dependent integration. All L1 interneurons tested exhibited direct responses to $\mathrm{Rh} \mathrm{TC}$ inputs, in agreement with the peak of $\mathrm{Rh}$ fiber density in this layer and as also reported for TC projections from diverse non-specific nuclei on L1 of the prefrontal cortex (Cruikshank et al. 2012). Given the weak responsiveness of L2/3 neurons to Rh inputs, this suggests that non-specific TC inputs favor inhibition of supragranular pyramidal neurons, which are a target of L1 interneurons (Chu et al. 2003, Wozny and Williams 2011). In contrast, we found that Rh TC inputs elicit robust and long-lasting responses of infragranular layers in the parietal association cortex. L6 contains pyramidal neurons that either send cortico-cortical projections or send inputs to the thalamic nuclei (Zhang and Deschenes 1997, Mercer et al. 2005, Kumar and Ohana 2008). Although we did not evaluate the relative strength or Rh inputs onto these two types of L6 pyramidal cells, it is likely that the output of cortico-thalamic cells is influenced by Rh input stimulation, either directly or via local recurrent excitation. We thus propose that the different non-specific thalamic nuclei strongly influence the input-output function of infragranular layers in their cognate cortical projection areas according to different sets of environmental and behavioral parameters.

\section{Rh TC inputs preferentially target Ad interneurons}

We show that the differential targeting of infragranular FS and Ad interneurons by Rh and VB TC inputs results in contrasting network dynamics, due to the different time constants (both intrinsic and synaptic) of these interneurons. FS cells are the largest cortical interneuron population, accounting for half of all interneurons in L6 (Perrenoud et al. 2013) and are the major interneuron target of VB TC inputs in the parietal association cortex, as reported in primary sensory areas (Agmon and Connors 1992, Gabernet et al. 2005, Cruikshank et al. 2007, Ji et al. 2016). Conversely, FS interneurons receive only weak Rh TC inputs, which strongly 
target Ad interneurons. Given the broad distribution of Rh TC fibers throughout L6, it is unlikely that their preferential targeting of Ad interneurons results from the reportedly skewed intralaminar density of FS interneurons in favor of upper L6 (Perrenoud et al. 2013). We found that the majority of infragranular Ad interneurons receive direct Rh TC inputs, suggesting that these inputs target several Ad interneuron types. Indeed, our results indicate that $\mathrm{Rh}$ TC inputs to L6 target both NPY and SOM interneuron types, which closely resemble cognate interneuron types found in other cortical layers (Karagiannis et al. 2009, Perrenoud et al. 2013). It is noteworthy that neurogliaform cells, the major morphological subtype of NPY interneurons (Karagiannis et al. 2009, Zaitsev et al. 2009, Perrenoud et al. 2013), and SOM interneurons both powerfully inhibit FS interneurons, whereas FS-to-Ad connections are weaker (Olah et al. 2007, Chittajallu et al. 2013, Cottam et al. 2013, Pfeffer et al. 2013, Xu et al. 2013, Jiang et al. 2015). These connections substantiate a critical feature of our model that enables Ad interneurons to dampen FS cell excitation upon Rh input stimulation, thereby resulting in longlasting responses of the network. Ad interneurons are generally more susceptible to the influence of various neuromodulators than FS interneurons (Bacci et al. 2005). This suggests that responses to Rh inputs may vary to a greater extent as a function of behavioral states than responses to primary sensory inputs. Moreover, Ad interneuron types differ in their activation time constants and sensitivity to neuromodulators (Cauli et al. 1997, Bacci et al. 2005, Ascoli et al. 2008, Karagiannis et al. 2009, Perrenoud et al. 2013). Hence, the dual targeting of NPY and SOM Ad interneuron types may enhance the integrative capability of Rh TC circuits by expanding the range of their cortical response durations and oscillatory behaviors.

\section{Cortical response dynamics depend on TC target interneuron type}

We demonstrate that differential targeting between FS and Ad interneurons is the key determinant of the different duration of cortical responses to $\mathrm{VB}$ and $\mathrm{Rh}$ inputs. The brief 
cortical responses to FS-targeting sensory TC inputs are well adapted to the detection of sensory stimuli with high temporal fidelity. Conversely, non-specific thalamic inputs convey information on the behavioral and environmental context that likely relies on persistent activity in cortical circuits. We found that $\mathrm{Rh}$ inputs induce a slowly decaying recurrent excitation, which forms a mnesic trace of past activation on a time scale relevant to behavior and may thus underlie cortical integration of contextual information. This TC response dichotomy may play a role in the flexible processing of context-relevant sensory signals by the parietal cortex (Ibos and Freedman 2014). Furthermore, our simulations indicate that targeting of Ad neurons by Rh inputs prolongs the cortical response and allows extended integration of VB and Rh inputs. Accordingly, neurons of the parietal cortex display near-perfect time integration of brief sensory signals (Huk and Shadlen 2005). Our model suggests that these temporal properties mainly depend on the state of Ad interneurons, which may underlie "state-dependent computations" (Buonomano and Maass 2009), i.e. information processing that depends on the internal dynamic state of the cortical network. We also give support to the computational hypothesis, according to which attentional effects would be mediated by interneurons with slow time constants (Buia and Tiesinga 2008). Indeed, we recently reported that responses to Rh inputs (targeting Ad interneurons) effectively integrate the effects of hypothalamic orexins, which signal vigilance states, on the cortical network (Hay et al. 2014).

In conclusion, our results show that target choice between slow- and fast-responding interneurons endows cortical circuits with a simple and powerful computational solution to perform both sensory detection and integration. A recent study by Audette and collaborators (2017) reports that higher-order somatosensory inputs from the posterior medial thalamic nucleus target fast or slow interneuron subtypes depending on the recipient cortical layer (L4 or L2). Here we show that non-specific thalamic inputs activate slow interneurons and that, in 
L6, both fast and slow integration can happen locally in the same micro-circuit. The coexistence of neurochemically distinct slow- and fast-responding inhibitory neurons is assessed in multiple regions of the central nervous system (Kawaguchi et al. 1995, Sosulina et al. 2010, Yasaka et al. 2010) and thus appears to be a widespread built-in property of neural networks. This suggests that the differential targeting of inhibitory neuron populations by incoming projection pathways can implement two complementary computations, detection and integration, in local microcircuits throughout the central nervous system. 


\section{REFERENCES}

Agmon A, Connors BW. 1992. Correlation between intrinsic firing patterns and thalamocortical synaptic responses of neurons in mouse barrel cortex. J Neurosci. 12:319-329.

Ascoli GA, Alonso-Nanclares L, Anderson SA, Barrionuevo G, Benavides-Piccione R, Burkhalter A, Buzsáki G, Cauli B, Defelipe J, Fairén A, Feldmeyer D, Fishell G, Fregnac Y, Freund TF, Gardner D, Gardner EP, Goldberg JH, Helmstaedter M, Hestrin S, Karube F, Kisvárday ZF, Lambolez B, Lewis DA, Marin O, Markram H, Muñoz A, Packer A, Petersen CC, Rockland KS, Rossier J, Rudy B, Somogyi P, Staiger JF, Tamas G, Thomson AM, Toledo-Rodriguez M, Wang Y, West DC, Yuste R. 2008. Petilla terminology: nomenclature of features of GABAergic interneurons of the cerebral cortex. Nat Rev Neurosci. 9:557-568.

Audette NJ, Urban-Ciecko J, Matsushita M, Barth AL. 2017. POm thalamocortical input drives layerspecific microcircuits in somatosensory cortex. Cer Cortex. 10:1:17.

Bacci A, Huguenard JR, Prince DA. 2005. Modulation of neocortical interneurons: extrinsic influences and exercises in self-control. Trends Neurosci. 28:602-610.

Bannister NJ, Benke TA, Mellor J, Scott H, Gürdal E, Crabtree JW, Isaac JT. 2005. Developmental changes in AMPA and kainate receptor-mediated quantal transmission at thalamocortical synapses in the barrel cortex. J Neurosci. 25:5259-5271.

Bayer L, Eggermann E, Saint-Mleux B, Machard D, Jones B, Mühlethaler M, Serafin M. 2002. Selective action of orexin (hypocretin) on nonspecific thalamocortical projection neurons. J Neurosci. 22:7835-7839.

Beierlein M, Gibson JR, Connors BW. 2003. Two dynamically distinct inhibitory networks in layer 4 of the neocortex. J Neurophysiol. 90:2987-3000.

Berendse HW, Groenewegen HJ. 1991. Restricted cortical termination fields of the midline and intralaminar thalamic nuclei in the rat. Neuroscience. 42:73-102.

Binshtok AM, Fleidervish IA, Sprengel R, Gutnick MJ. 2006. NMDA receptors in layer 4 spiny stellate cells of the mouse barrel cortex contain the NR2C subunit. J Neurosci. 26:708-715.

Brunel N, Wang XJ. 2003. What determines the frequency of fast network oscillations with irregular neural discharges? I. Synaptic dynamics and excitation-inhibition balance. J Neurophysiol. 90:415430.

Buia CI, Tiesinga PH. 2008. Role of interneuron diversity in the cortical microcircuit for attention. J Neurophysiol. 99:2158-2182.

Buonomano DV, Maass W. 2009. State-dependent computations: spatiotemporal processing in cortical networks. Nat Rev Neurosci. 10:113-125.

Cauli B, Audinat E, Lambolez B, Angulo MC, Ropert N, Tsuzuki K, Hestrin S, Rossier J. 1997. Molecular and physiological diversity of cortical nonpyramidal cells. J Neurosci. 17:3894-3906. 
Chittajallu R, Pelkey KA, McBain CJ. 2013. Neurogliaform cells dynamically regulate somatosensory integration via synapse-specific modulation. Nat Neurosci. 16:13-15.

Cholvin T, Loureiro M, Cassel R, Cosquer B, Geiger K, De Sa Nogueira D, Raingard H, Robelin L, Kelche C, Pereira de Vasconcelos A, Cassel JC. 2013. The ventral midline thalamus contributes to strategy shifting in a memory task requiring both prefrontal cortical and hippocampal functions. $\mathbf{J}$ Neurosci. 33:8772-8783.

Chu Z, Galarreta M, Hestrin S. 2003. Synaptic interactions of late-spiking neocortical neurons in layer 1. J Neurosci. 23:96-102.

Constantinople CM, Bruno RM. 2013. Deep cortical layers are activated directly by thalamus. Science. 340:1591-1594.

Cottam JC, Smith SL, Häusser M. 2013. Target-specific effects of somatostatin-expressing interneurons on neocortical visual processing. J Neurosci. 33:19567-19578.

Crunelli V, Kelly JS, Leresche N, Pirchio M. 1987. On the excitatory post-synaptic potential evoked by stimulation of the optic tract in the rat lateral geniculate nucleus. J Physiol. 384:603-618.

Cruikshank SJ, Lewis TJ, Connors BW. 2007. Synaptic basis for intense thalamocortical activation of feedforward inhibitory cells in neocortex. Nat Neurosci. 10:462-468.

Cruikshank SJ, Urabe H, Nurmikko AV, Connors BW. 2010. Pathway-specific feedforward circuits between thalamus and neocortex revealed by selective optical stimulation of axons. Neuron. 65:230-245.

Cruikshank SJ, Ahmed OJ, Stevens TR, Patrick SL, Gonzalez AN, Elmaleh M, Connors BW. 2012. Thalamic control of layer 1 circuits in prefrontal cortex. J Neurosci. 32:17813-17823.

Dayan P, Abbott LF. 2001. Network models. In: Dayan P, Abbott LF, editors. Theoretical neuroscience. Vol 1. Cambridge (MA, USA): The MIT press. p 1-150.

Destexhe A, Mainen ZF, Sejnowski TJ. 1998. Kinetic models of synaptic transmission. In: Koch C, Segev I, editors. Methods in Neuronal Modeling. Cambridge (MA, USA): The MIT press. p 1-25.

Feldmeyer D, Egger V, Lubke J, Sakmann B. 1999. Reliable synaptic connections between pairs of excitatory layer 4 neurones within a single "barrel" of developing rat somatosensory cortex. J Physiol. 521:169-190.

Gabernet L, Jadhav SP, Feldman DE, Carandini M, Scanziani M. 2005. Somatosensory integration controlled by dynamic thalamocortical feed-forward inhibition. Neuron. 48:315-327.

Geiger JR, Lübke J, Roth A, Frotscher M, Jonas P. 1997. Submillisecond AMPA receptor-mediated signaling at a principal neuron-interneuron synapse. Neuron. 6:1009-1023.

Gil Z, Amitai Y. 1996. Adult thalamocortical transmission involves both nmda and non-nmda receptors. J Neurophysiol. 76:2547-2554.

Gil Z, Connors BW, Amitai Y. 1999. Efficacy of thalamocortical and intracortical synaptic connections: quanta, innervation, and reliability. Neuron. 23:385-397. 
Goel A, Buonomano DV. 2014. Timing as an intrinsic property of neural networks: evidence from in vivo and in vitro experiments. Philos Trans R Soc Lond B Biol Sci. 369:20120460.

Goldberg EM, Jeong HY, Kruglikov I, Tremblay R, Lazarenko RM, Rudy B. 2011. Rapid developmental maturation of neocortical FS cell intrinsic excitability. Cereb Cortex. 21:666-682.

Hallock HL, Wang A, Shaw CL, Griffin AL. 2013. Transient inactivation of the thalamic nucleus reuniens and rhomboid nucleus produces deficits of a working-memory dependent tactile-visual conditional discrimination task. Behav Neurosci. 127:860-866.

Hartveit E, Veruki ML. 2007. Studying properties of neurotransmitter receptors by non-stationary noise analysis of spontaneous postsynaptic currents and agonist-evoked responses in outside-out patches. Nat. Prot. 2:434-448.

Hay YA, Andjelic S, Badr S, Lambolez B. 2014. Orexin-dependent activation of layer VIb enhances cortical network activity and integration of non-specific thalamocortical inputs. Brain Struct Funct. 220:3497-3512.

Hembrook JR, Mair RG. 2011. Lesions of reuniens and rhomboid thalamic nuclei impair radial maze win-shift performance. Hippocampus. 21:815-826.

Herkenham M. 1980. Laminar organization of thalamic projections to the rat neocortex. Science. 207:532-535.

Hildebrand ME, Pitcher GM, Harding EK, Li H, Beggs S, Salter MW. 2014. GluN2B and GluN2D NMDARs dominate synaptic responses in the adult spinal cord. Sci Rep. 4:4094.

Hu H, Martina M, Jonas P. 2010. Dendritic mechanisms underlying rapid synaptic activation of fastspiking hippocampal interneurons. Science. 327:52-58.

Huk AC, Shadlen MN. 2005. Neural activity in macaque parietal cortex reflects temporal integration of visual motion signals during perceptual decision making. J Neurosci. 25:10420-10436.

Hull C, Adesnik H, Scanziani M. 2009. Neocortical disynaptic inhibition requires somatodendritic integration in interneurons. J Neurosci. 29:8991-8995.

Ibos G, Freedman DJ. 2014. Dynamic integration of task-relevant visual features in posterior parietal cortex. Neuron. 83:1468-1480.

Ito HT, Zhang SJ, Witter MP, Moser EI, Moser MB. 2015. A prefrontal-thalamo-hippocampal circuit for goal-directed spatial navigation. Nature. 522:52-55.

Jahnsen H, Llinás R. 1984. Electrophysiological properties of guinea-pig thalamic neurons: an in vitro study. J Physiol. 349:205-226.

Jasper H, Ajmone-Marsan C, Hanbery J. 1953. Pathways and functional properties of the nonspecific thalamo-cortical projection system. Trans Am Neurol Assoc. 3:9-11.

Ji XY, Zingg B, Mesik L, Xiao Z, Zhang LI, Tao HW. 2016. Thalamocortical Innervation Pattern in Mouse Auditory and Visual Cortex: Laminar and Cell-Type Specificity. Cereb Cortex. 26:26122625 . 
Jiang X, Shen S, Cadwell CR, Berens P, Sinz F, Ecker AS, Patel S, Tolias AS. 2015. Principles of connectivity among morphologically defined cell types in adult neocortex. Science. 350:aac9462.

Jones E. 2007. Principles of thalamic organization. In: Jones E, editor. The Thalamus. 2nd ed. Cambridge (UK): Cambridge University Press. p 87-170.

Kapfer C, Glickfeld LL, Atallah BV, Scanziani M. 2007. Supralinear increase of recurrent inhibition during sparse activity in the somatosensory cortex. Nat Neurosci. 6:743-753.

Karagiannis A, Gallopin T, Dávid C, Battaglia D, Geoffroy H, Rossier J, Hillman EM, Staiger JF, Cauli B. 2009. Classification of NPY-expressing neocortical interneurons. J Neurosci. 29:3642-3659.

Kawaguchi Y. 1995. Physiological subgroups of nonpyramidal cells with specific morphological characteristics in layer II/III of rat frontal cortex. J Neurosci. 15:2638-2655.

Kawaguchi Y, Wilson CJ, Augood SJ, Emson PC. 1995. Striatal interneurons: chemical, physiological and morphological characterization. Trends Neurosci. 18:527-535.

Kumar P, Ohana O. 2008. Inter- and intralaminar subcircuits of excitatory and inhibitory neurons in layer 6a of the rat barrel cortex. J Neurophysiol. 100:1909-1922.

Loureiro M, Cholvin T, Lopez J, Merienne N, Latreche A, Cosquer B, Geiger K, Kelche C, Cassel JC, Pereira de Vasconcelos A. 2012. The ventral midline thalamus (reuniens and rhomboid nuclei) contributes to the persistence of spatial memory in rats. J Neurosci. 32:9947-9959.

Maskos U, Molles BE, Pons S, Besson M, Guiard BP, Guilloux JP, Evrard A, Cazala P, Cormier A, Mameli-Engvall M, Dufour N, Cloëz-Tayarani I, Bemelmans AP, Mallet J, Gardier AM, David V, Faure P, Granon S, Changeux JP. 2005. Nicotine reinforcement and cognition restored by targeted expression of nicotinic receptors. Nature. 436:103-107.

Mercer A, West DC, Morris OT, Kirchhecker S, Kerkhoff JE, Thomson AM. 2005. Excitatory connections made by presynaptic cortico-cortical pyramidal cells in layer 6 of the neocortex. Cereb Cortex. 15:1485-1496.

Meyer HS, Wimmer VC, Hemberger M, Bruno RM, de Kock CPJ, Frick A, Sakmann B, Helmstaedter M. 2010. Cell type-specific thalamic innervation in a column of rat vibrissal cortex. Cereb Cortex. 20:2287-2303.

Moruzzi G, Magoun HW. 1949. Brain stem reticular formation and activation of the eeg. Electroencephalogr Clin Neurophysiol. 1:455-473.

Oláh S, Komlósi G, Szabadics J, Varga C, Tóth E, Barzó P, Tamás G. 2007. Output of neurogliaform cells to various neuron types in the human and rat cerebral cortex. Front Neural Circuits. 1:4.

Osten P, Dittgen T, Licznerski P. 2006. Lentivirus-based genetic manipulations in neurons In Vivo. In: Kittler JT, Moss SJ, editors. The Dynamic synapse: Molecular Methods in Ionotropic Receptor Biology. Boca Raton (FL): CRC Press, ch13.

Paxinos G, Watson C. 2007. The Rat Brain in Stereotaxic Coordinates. London:(UK): Academic Press. Perrenoud Q, Rossier J, Geoffroy H, Vitalis T, Gallopin T. 2013. Diversity of GABAergic interneurons in layer VIa and VIb of mouse barrel cortex. Cereb Cortex. 23:423-441. 
Peters A, Jones EG. 1984. Classification of cortical neurons. In: Peters A, Jones EG, editors. Cerebral Cortex: Cellular Components of the Cerebral Cortex. New York (NY, USA): Plenum. p 107-121.

Petreanu L, Mao T, Sternson SM, Svoboda K. 2009. The subcellular organization of neocortical excitatory connections. Nature. 457:1142-1145.

Pfeffer CK, Xue M, He M, Huang ZJ, Scanziani M. 2013. Inhibition of inhibition in visual cortex: the logic of connections between molecularly distinct interneurons. Nat Neurosci. 16:1068-1076.

Porter JT, Johnson CK, Agmon A. 2001. Diverse types of interneurons generate thalamus-evoked feedforward inhibition in the mouse barrel cortex. J Neurosci. 21:2699-2710.

Saalmann YB. 2014. Intralaminar and medial thalamic influence on cortical synchrony, information transmission and cognition. Front Syst Neurosci. 9:1-8.

Sigworth FJ.1980. The variance of sodium current fluctuations at the node of Ranvier. J Physiol 307:97129

Sosulina L, Graebenitz S, Pape HC. 2010. GABAergic interneurons in the mouse lateral amygdala: a classification study. J Neurophysiol. 104:617-626.

Staiger JF, Flagmeyer I, Schubert D, Zilles K, Kötter R, Luhmann HJ. 2004. Functional diversity of layer IV spiny neurons in rat somatosensory cortex: quantitative morphology of electrophysiologically characterized and biocytin labeled cells. Cereb Cortex 14:690-701.

Swadlow HA. 2003. Fast-spike interneurons and feedforward inhibition in awake sensory neocortex. Cereb Cortex. 13:25-32.

Tan Z, Hu H, Huang ZJ, Agmon A. 2008. Robust but delayed thalamocortical activation of dendritictargeting inhibitory interneurons. Proc Natl Acad Sci U S A. 105:2187-2192.

Thomson AM, Bannister AP. 2003. Interlaminar connections in the neocortex. CerebCortex. 13:5-14.

Van der Werf YD, Witter MP, Groenewegen HJ. 2002. The intralaminar and midline nuclei of the thalamus. anatomical and functional evidence for participation in processes of arousal and awareness. Brain Res Brain Res Rev. 39:107-140.

Vertes RP, Hoover WB, Do Valle AC, Sherman A, Rodriguez JJ. 2006. Efferent projections of reuniens and rhomboid nuclei of the thalamus in the rat. J Comp Neurol. 499:768-796.

Wilson HR, Cowan JD. 1972. Excitatory and inhibitory interactions in localized populations of model neurons. Biophys J. 12:1-24.

Wimmer VC, Bruno RM, de Kock CP, Kuner T, Sakmann B. 2010. Dimensions of a projection column and architecture of VPM and POm axons in rat vibrissal cortex. Cereb Cortex. 20:2265-2276.

Wozny C, Williams SR. 2011. Specificity of synaptic connectivity between layer 1 inhibitory interneurons and layer 2/3 pyramidal neurons in the rat neocortex. Cereb Cortex. 21:1818-1826.

Xu H, Jeong H-Y, Tremblay R, Rudy B. 2013. Neocortical Somatostatin-expressing GABAergic Interneurons Disinhibit the Thalamorecipient Layer 4. Neuron. 77:155-167. 
Yasaka T, Tiong SY, Hughes DI, Riddell JS, Todd AJ. 2010. Populations of inhibitory and excitatory interneurons in lamina II of the adult rat spinal dorsal horn revealed by a combined electrophysiological and anatomical approach. Pain. 151:475-488.

Zaitsev AV, Povysheva NV, Gonzalez-Burgos G, Rotaru D, Fish KN, Krimer LS, Lewis DA. 2009. Interneuron diversity in layers 2-3 of monkey prefrontal cortex. Cereb Cortex. 19:1597-1615.

Zhang F, Aravanis AM, Adamantidis A, de Lecea L, Deisseroth K. 2007. Circuit-breakers: optical technologies for probing neural signals and systems. Nat Rev Neurosci. 8:577-581.

Zhang ZW, Deschênes M. 1997. Intracortical axonal projections of lamina VI cells of the primary somatosensory cortex in the rat: a single-cell labeling study. J Neurosci. 17:6365-6379. 


\section{FIGURE LEGENDS}

\section{Figure 1: Expression of $\mathrm{ChR2}$ in $\mathrm{Rh}$ and $\mathrm{VB}$ thalamic nuclei and in TC fibers}

(A-B) Whole-field fluorescence negative pictures showing expression of ChR2-YFP (black) in the $\mathrm{Rh}$ (delineated in red) and VB nuclei after viral transduction. The superimposed sections of the rat brain atlas delineate thalamic nuclei (CM: centromedian, Re: reuniens, Sub: submedius, Rt: reticular, Po: posterior complex, VPM: ventroposteromedian, VPL: ventroposterolateral). Note that the VB nucleus comprises VPM and VPL. (C) Combined infrared and fluorescence negative picture of a coronal slice showing ChR2-YFP+ Rh TC fibers (black) in the recording area of the parietal association cortex (PtA), located above the CA1 hippocampal subfield. (D) Left: Whole-field fluorescence pictures illustrating the distribution of DAPI staining and ChR2YFP+ Rh or VB TC fibers in coronal slices of the parietal association cortex. Right: Plots of mean fluorescence intensity across cortical layers for DAPI $(n=5), \operatorname{Rh}$ TC fibers $(n=5)$ and VB TC fibers ( $n=6)$. The peak of DAPI fluorescence corresponds to the highest density of neurons, which occurs in L4, and coincides with the main peak of VB fibers density. The main peak of Rh fibers density is located in L6 and roughly coincides with the minor peak of VB fibers.

\section{Figure 2: Rh and VB TC inputs elicit network responses of contrasting duration}

(A) Confocal reconstruction of a recorded L6 pyramidal cell (red) surrounded by ChR2-YFP+ Rh TC fibers. Inset: Infrared picture of this neuron. (B) Responses of the same neuron as in (A) to current pulses. Photostimulation (blue bar) induced transient inward currents that were

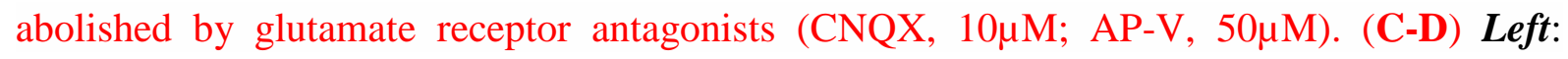
Superimposed traces of averaged and normalized EPSCs (C) and IPSCs (D) elicited in L6 pyramidal neurons by photostimulation of either Rh (black) or VB (grey) TC fibers. Right: 
plots of PSCs mean durations at one third of maximal amplitude (arrows on traces), and of mean values of parameters describing their shape and kinetics.

\section{Figure 3: Properties of the glutamatergic Rh TC synapse onto L6 pyramidal neurons}

(A) Distribution of EPSC delays-to-onset measured on 41 L6 pyramidal neurons at maximal photostimulation intensity. (B) Left and middle: Superimposed EPSCs normalized to the first peak (gray) and mean EPSC (black) obtained at different stimulus intensities. Right: delay-toonset and failure of direct EPSCs as a function of photostimulation intensity ( $\mathrm{n}=6$ neurons). (C)

Left: superimposition of direct EPSCs (grey traces) recorded at $+50 \mathrm{mV}$ (top, 10 events) and $70 \mathrm{mV}$ (bottom, 10 events). Averaged EPSCs (black) were used to measure the amplitudes of AMPAR- and NMDAR-mediated currents where indicated. Inset: Same traces at expanded time-scale. Middle: mean amplitude of AMPAR and NMDAR mediated currents and mean NMDA/AMPA ratio measured in 11 neurons. Right: AMPA and NMDA currents were abolished by their respective antagonists. (C-D) Single channel properties at the Rh TC synapse. (D) Example of non-stationary noise analysis of AMPAR-mediated synaptic currents in a single neuron. Upper panels: superimposition of direct EPSCs recorded at - $70 \mathrm{mV}$ (left) and distribution of direct EPSC amplitudes (right). Lower: the plot of the mean variance of the current as a function of the mean EPSC amplitude was fitted with a parabolic function, which allows the determination of the unitary conductance (y) of AMPAR channels mediating the EPSCs. Inset: Variance of the baseline current. (E) Same analysis as in (D) for the characterization of synaptic NMDAR single channel properties in a neuron recorded at +50 $\mathrm{mV}$.

Figure 4: Optogenetic mapping of the cortical targets of Rh neurons 
(A) Light-induced responses were blocked by TTX $(1 \mu \mathrm{M})$ but recovered in the additional presence of 4AP $(1 \mathrm{mM})$. Note that TTX-4AP converted polyphasic responses into essentially monophasic ones, but markedly slowed down EPSC kinetics. (B) Recurrent excitation and inhibition were blocked by TTX and 4AP, while the amplitude of direct excitation was roughly similar in control and TTX-4AP conditions. (C) Left: Composite confocal reconstruction of a strip of parietal association cortical slice showing ChR2-expressing Rh TC fibers (green) in all layers and neurons (red) recorded in the presence of TTX and 4AP. Recorded cells were identified according to dendritic spine density as excitatory neurons (Pyr, high density) or interneurons (IN, low density). The sparsely spiny L2/3 cell was classified as Ad interneuron based on its absence of parvalbumin (PV) immunolabeling, whereas the PV+ aspiny cell in L6 was classified as an FS interneuron. Right: Direct EPSCs recorded in these neurons upon photostimulation of Rh fibers in the presence of TTX and 4AP. (D) Mean amplitude of direct EPSCs recorded in the presence of TTX-4AP in neurons across L1-6, and proportion of these neurons showing no response to photostimulation. Note that neuron responsiveness roughly followed the mean fluorescence intensity profile of $\mathrm{Rh}$ fibers (green, $\mathrm{n}=5$ slices) and was minimal in L4.

Figure 5: Rh and VB TC inputs exhibit opposite preference for Ad and FS interneurons (A) Left: Examples of Rh and VB TC EPSCs recorded in TTX-4AP conditions in infragranular pyramidal, Ad and FS neurons. Right: Mean amplitudes of direct Rh and VB TC EPSCs in the three neuron types. (B) From left to right: Biocytin labeling and immunohistochemistry revealed expression of neuropeptide Y (NPY) or somatostatin (SOM) in L6 Ad interneurons responsive to $\mathrm{Rh}$ TC inputs. Responses of the same interneurons to the injection of hyperpolarising and depolarising current pulses (40 pA), and to photostimulation of $\mathrm{Rh} \mathrm{TC}$ fibers in the presence of TTX-4AP. SOM was detected in 12 out of 91 responsive interneurons 
and NPY in 11 out of 21 responsive interneurons tested. Mean amplitude of EPSCs recorded in 12 SOM-positive and 11 NPY-positive Ad interneurons.

Figure 6: Rh and VB TC inputs differentially recruit FS and Ad interneurons and elicit different temporal sequences of excitation/inhibition

(A) Examples of FS and Ad interneurons activation and mean delay-to-spike upon Rh or VB fiber photostimulation. Note that action potential (AP) discharge in FS interneurons is delayed upon Rh stimulation compared to VB stimulation and that VB stimulation does not induce firing of Ad interneurons. (B) Post-stimulus latencies of EPSCs and IPSCs onsets in pyramidal neurons. Note that current amplitudes have been normalized to highlight the similarity of EPSC onset kinetics and the different delay-to-onset of IPSCs elicited by VB or Rh stimulation. (C) Plots summarizing delays-to-onset of EPSCs and IPSCs in pyramidal neurons and delays-tospike in FS and Ad interneurons for the two thalamocortical pathways. Upon VB stimulation, EPSC, FS firing and IPSC are arranged sequentially in a narrow time-window. Upon Rh stimulation, events occur in broad time-window and their distributions largely overlap. The cartoons summarize the main features of the two thalamocortical circuits identified in this study, and incorporate cortico-cortical connections described in the literature (see main text).

Figure 7: Differential activation of FS and Ad interneurons leads to distinct time-courses of TC response in a mean field model

The cartoons depict the thalamocortical circuits used to build mean-field models that predict the firing rates of the neuronal populations as a function of their intrinsic properties, cortical connectivity and thalamic inputs. (A) A reduced model comprising only an excitatory and an inhibitory population predicts that the system is unstable below a certain inhibitory feedback, but otherwise returns to its initial state (stable) with a characteristic time constant that depends 
on the properties of inhibitory neurons. The decay of the response is monotonous or oscillatory depending on interneuron properties and their coupling with excitatory neurons. (B) Analyses of a detailed mean-field model comprising the three pyramidal, Ad and FS neuronal populations. Upper panels: Examples of responses of the model following Rh or VB input stimulation (light blue bars, 1ms). Note the different time-courses of pyramidal neuron population activity elicited by VB and Rh inputs. Lower panels: Evolution of pyramidal cell activity elicited by VB or Rh inputs during the $100 \mathrm{~ms}$ post-stimulus period. (C) Rh inputs on Ad interneurons shape the duration of the cortical network activation. Central panel: Suppression of Rh inputs on Ad interneurons (represented by the scissors on the cartoon) reduces the duration of the cortical activation for all three neuronal populations due to a prominent feedback recruitment of FS neurons. Right: Duration of pyramidal neurons activity increases as a function of Rh input strength on Ad cells. (D) Right: examples of responses to successive Rh and VB input stimulation in conditions where Rh targets either both pyramidal and Ad neurons (Pyr + Ad, black) or only pyramidal cells (Pyr only, grey). Left: peak amplitude of the response to VB input stimulation as a function of time after Rh stimulation. 


\section{SUPPLEMENTARY FIGURE LEGENDS}

\section{Supplementary figure 1: Expression of ChR2 in Rh thalamic neurons}

(A) Whole-field fluorescence negative pictures of serial brain sections along the anteroposterior axis (AP, relative to Bregma) showing expression of ChR2-YFP (black) in the Rh nucleus 4 weeks after viral transduction in a P12 rat. The viral injection site is between AP - 2.8 and AP 3.3 sections. The superimposed sections of the rat brain atlas delineates thalamic nuclei (CM: centromedian, Re: reuniens, PVP: paraventricular nucleus posterior part, Sub: submedius). Note that the intense black stripe adjacent to the YFP-labeled Rh nucleus on picture AP -3.8 $\mathrm{mm}$ is an artifact due to damage in this section. (B) Combined infrared and fluorescence picture showing another example of ChR2-YFP expression (green) in the Rh nucleus after viral transduction. (C) Confocal reconstruction (left: $\mathrm{z}$-stack, right: single plane) of a recorded $\mathrm{Rh}$ neuron (red). Note that ChR2-EYFP is present at the plasma membrane of the recorded neuron. (D) Responses of a ChR2-expressing Rh neuron to depolarizing and hyperpolarizing current steps (left) and to photostimulation (1 ms pulses, blue bars, middle and right panels). (E) Application of orexin B depolarizes ChR2-expressing Rh neurons and converts responses to photostimulation into a tonic discharge of action potentials.

\section{Supplementary figure 2: Morphology of recorded neurons}

(A) Examples of confocal reconstruction (z-stack) of excitatory or inhibitory neurons (red) recorded in indicated cortical layers and of ChR2-YFP labeled fibers (green). Note that L1 is devoid of excitatory neurons. (B) Examples of confocal reconstruction and immunostaining of biocytin-filled FS and Ad interneurons recorded in L6 (PV: parvalbumin, NPY: neuropeptide Y, SOM: somatostatin) and bar graphs summarizing somatodendritic morphologies observed 
in L6 SOM+ and NPY+ Ad interneurons. SOM+ Ad interneurons exhibited bipolar/bitufted (upper middle panel) or multipolar (upper right panel) morphologies with thick primary dendrites that rarely branched. A majority of NPY+ Ad interneurons were also bipolar/bitufted or multipolar (lower right panel), but some exhibited neurogliaform-like morphology with thin and ramified dendrites (lower middle panel).

\section{Supplementary figure 3: Electrophysiological properties of FS and Ad interneurons}

Upper panels: Biocytin (Bio) labeling of recorded FS and Ad interneurons. The FS interneuron is parvalbumine (PV) positive and the Ad interneuron is PV-. Traces show spontaneous EPSCs recorded at a holding potential of $-70 \mathrm{mV}$ in these interneurons. The bar graphs summarize EPSC frequency recorded using K-gluconate or Cs-gluconate intracellular solution in FS neurons ( $n=13$ and $n=6$, respectively) or Ad neurons ( $n=18$ and $n=6$, respectively). Upper middle panel: Firing properties of FS $(n=13)$ and $\operatorname{Ad}(n=18)$ neurons in control conditions. Lower panels: Responses to current injection recorded using K-gluconate intracellular solution in the presence of TTX $(1 \mu \mathrm{M})$ and 4AP $(1 \mathrm{mM})$ in FS $(\mathrm{n}=10)$ and $\mathrm{Ad}(\mathrm{n}=10)$ neurons or using Cs-gluconate intracellular solution in control conditions in FS ( $n=6)$ and $\mathrm{Ad}(\mathrm{n}=6)$ interneurons.

\section{Supplementary figure 4: Onset slopes of EPSCs and action potentials in FS and Ad interneurons}

(A) The 20-80\% rise time of short-latency (direct) EPSCs recorded upon Rh TC stimulation in control conditions is twice slower in $\mathrm{Ad}(73 \pm 34 \mathrm{pA} / \mathrm{ms}, \mathrm{n}=9)$ than in FS interneurons $(168 \pm 64$ $\mathrm{pA} / \mathrm{ms}, \mathrm{n}=19, \mathrm{p}<0.05)$. (B) Action potential rise time following Rh TC stimulation is slower in $\operatorname{Ad}(0.88 \pm 0.04 \mathrm{~ms}, \mathrm{n}=18)$ than in FS interneurons $(0.56 \pm 0.03 \mathrm{~ms}, \mathrm{n}=14, \mathrm{p}<0.05)$. Rise time is measured from the initiation to the peak of the action potential (black bars). 
Supplementary figure 5: Differential activation of FS and Ad interneurons leads to contrasting cortical network dynamics in a mean-field model

(A) Oscillation frequency in the reduced model of excitatory-inhibitory interactions. Null frequencies (black) correspond to non-oscillatory behaviors. (B) Vector fields depicting phase plane portraits of the activity of pyramidal cells and FS or Ad interneurons in the detailed model. Initial strength of VB (mainly activating pyramidal and FS cells, top) and Rh (mainly activating pyramidal and Ad cells, bottom) thalamic inputs on neuronal populations are given by the locations of the arrows. Size of the arrows indicates the speed of the return to initial state. (C) Responses of the pyramidal cell population as a function of $\mathrm{Rh}$ input strength on pyramidal and Ad cells in the detailed model. 


\section{Figure 1}
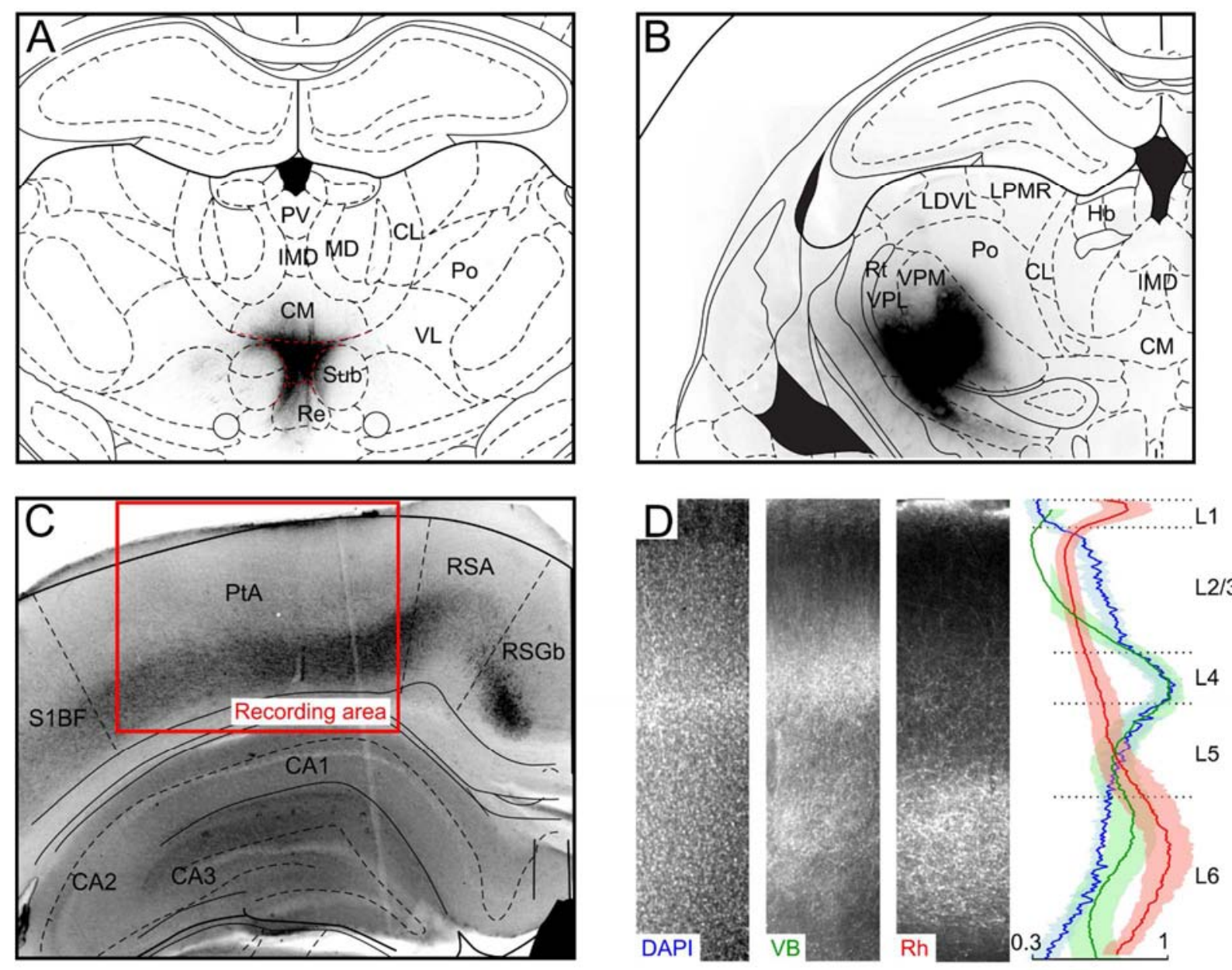

Fluorescence intensity (relative to peak) 


\section{Figure 2}
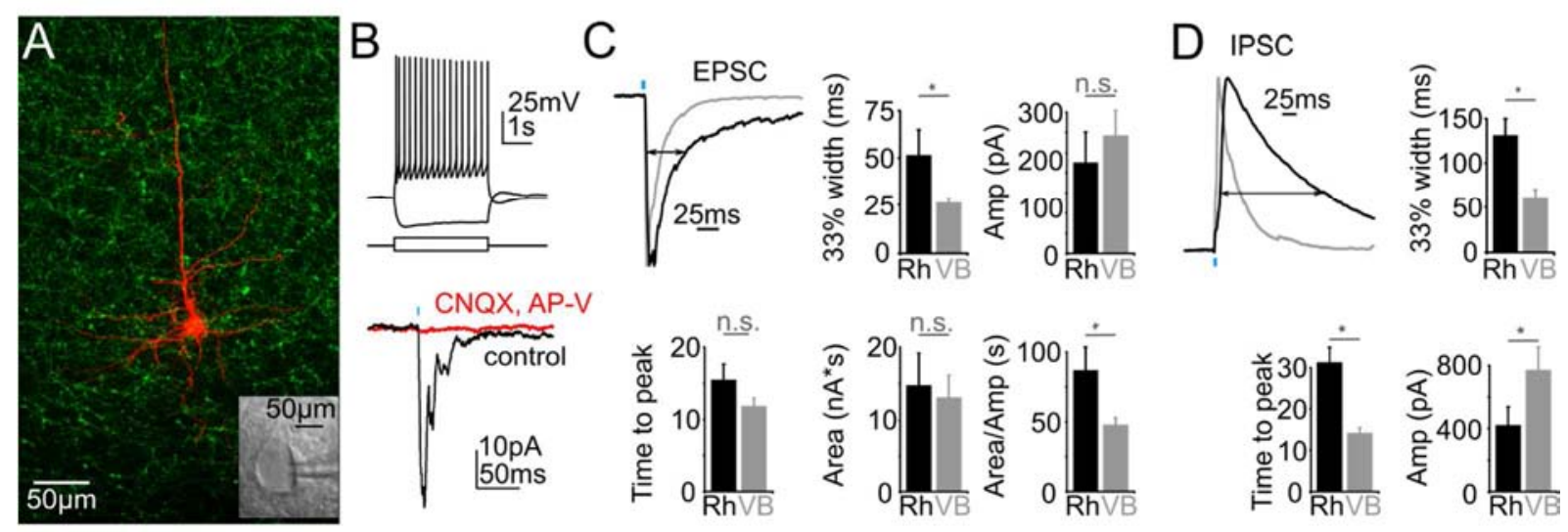


\section{Figure 3}
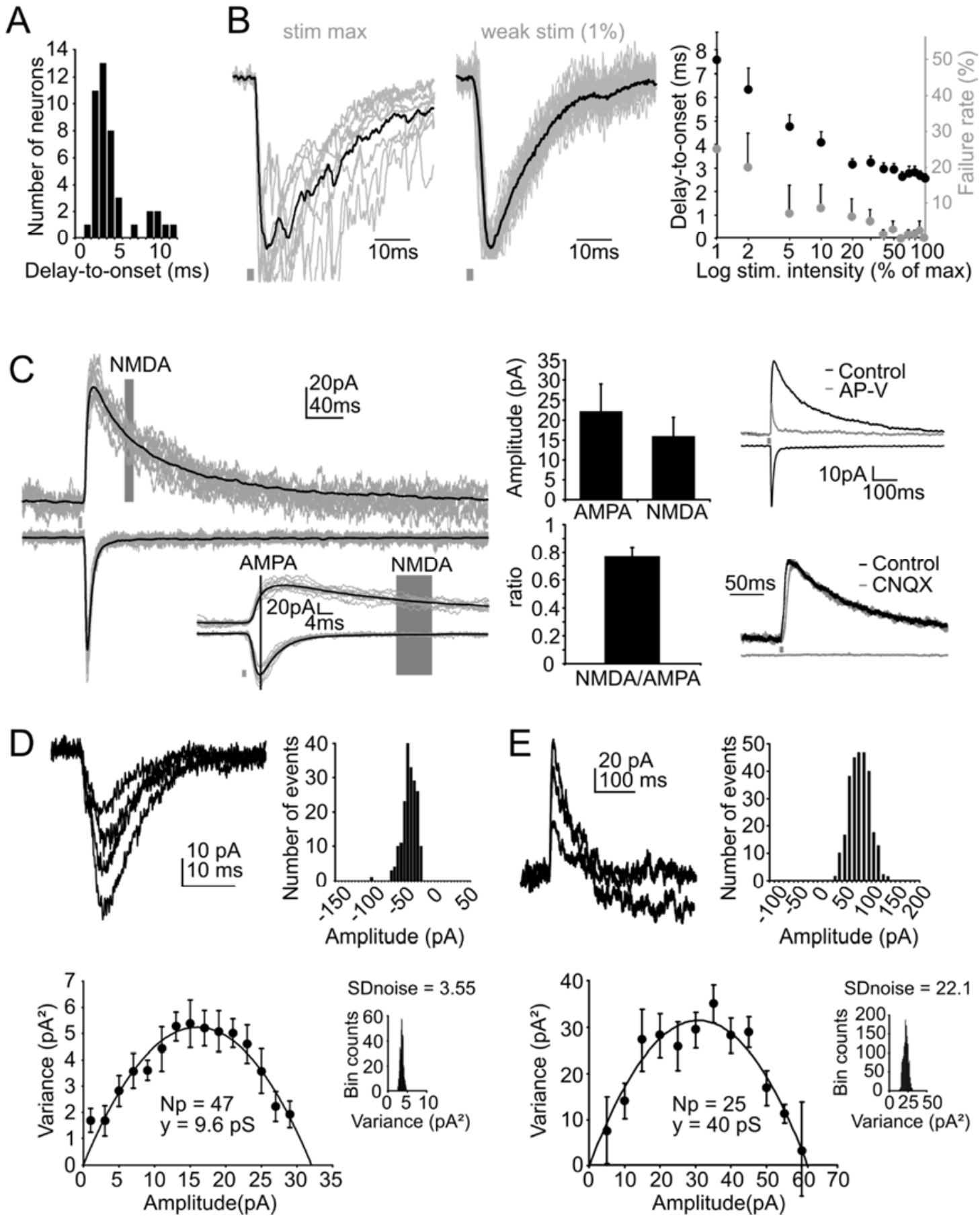


\section{Figure 4}

A
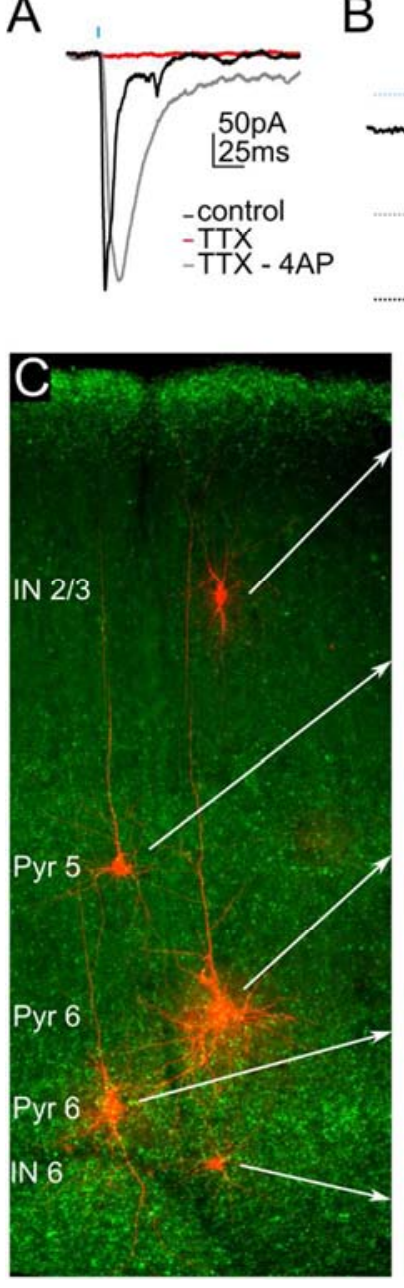

B TTX-4AP
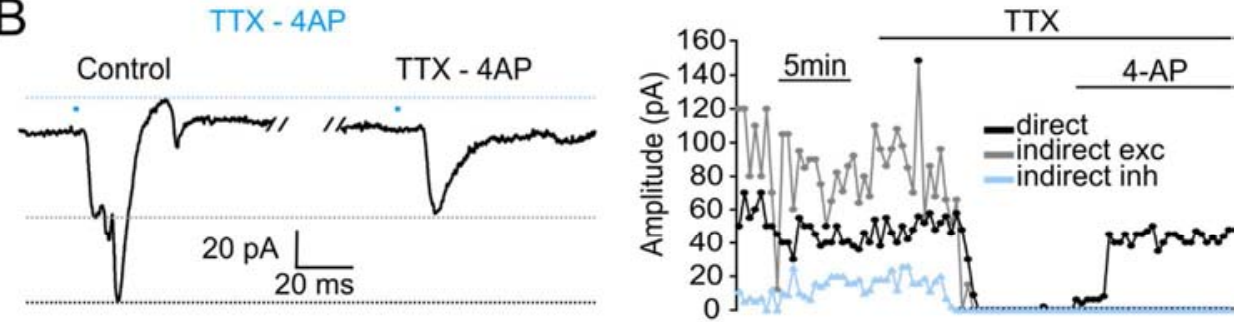

D

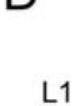

L2/3

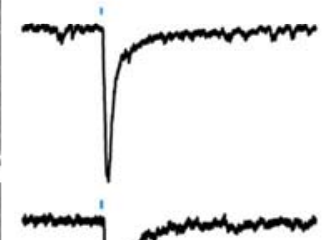

50pA

$200 \mathrm{~ms}$
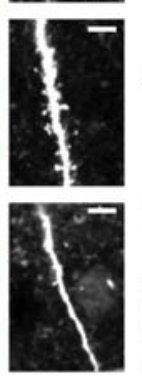

\section{EPSC amplitude (pA)}

L1

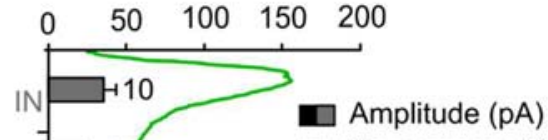

L4

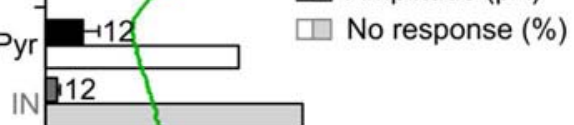

L5

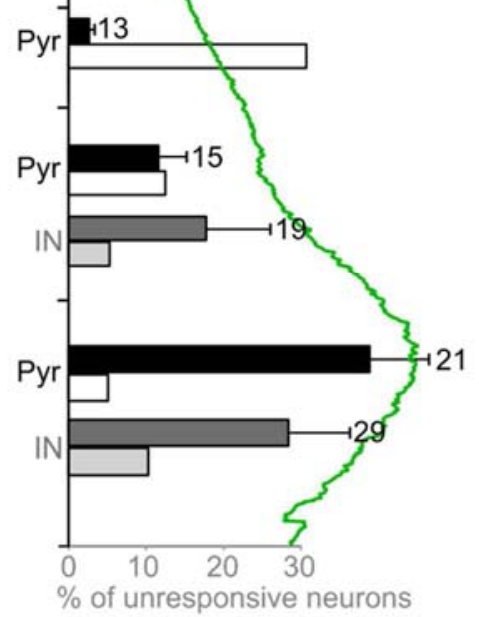

$\%$ of unresponsive neurons
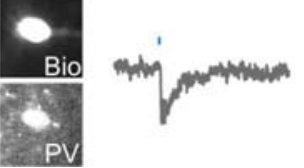


\section{Figure 5}

A Rh/VB - TTX-4AP
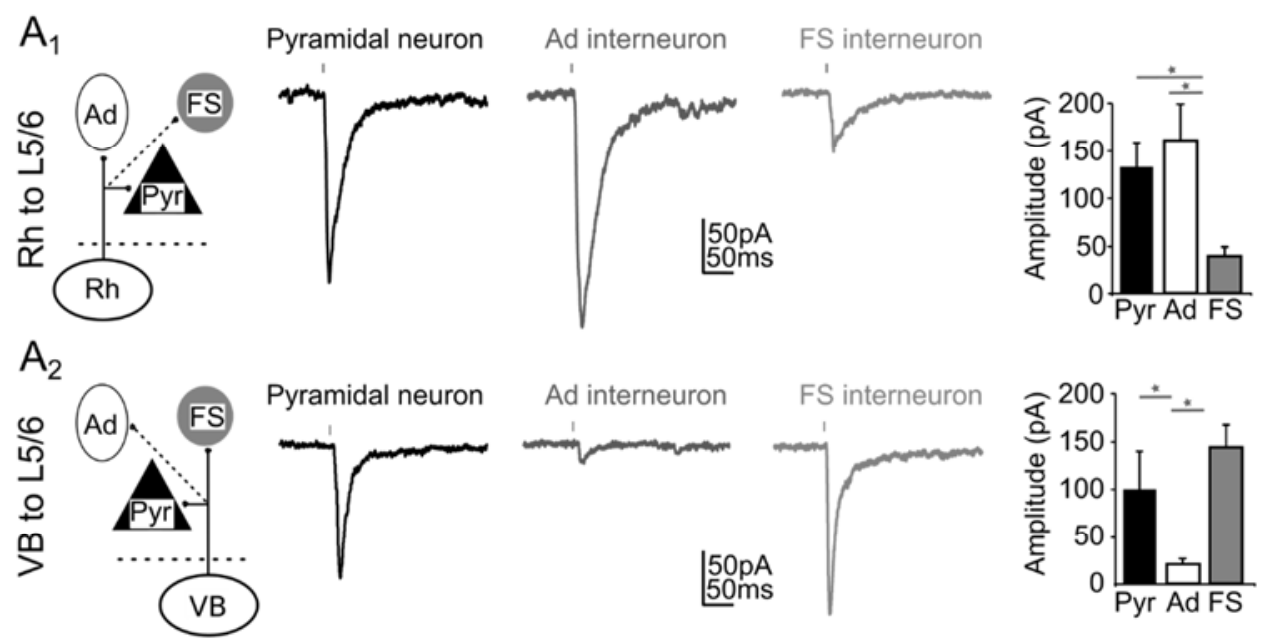

B Rh - TTX-4AP
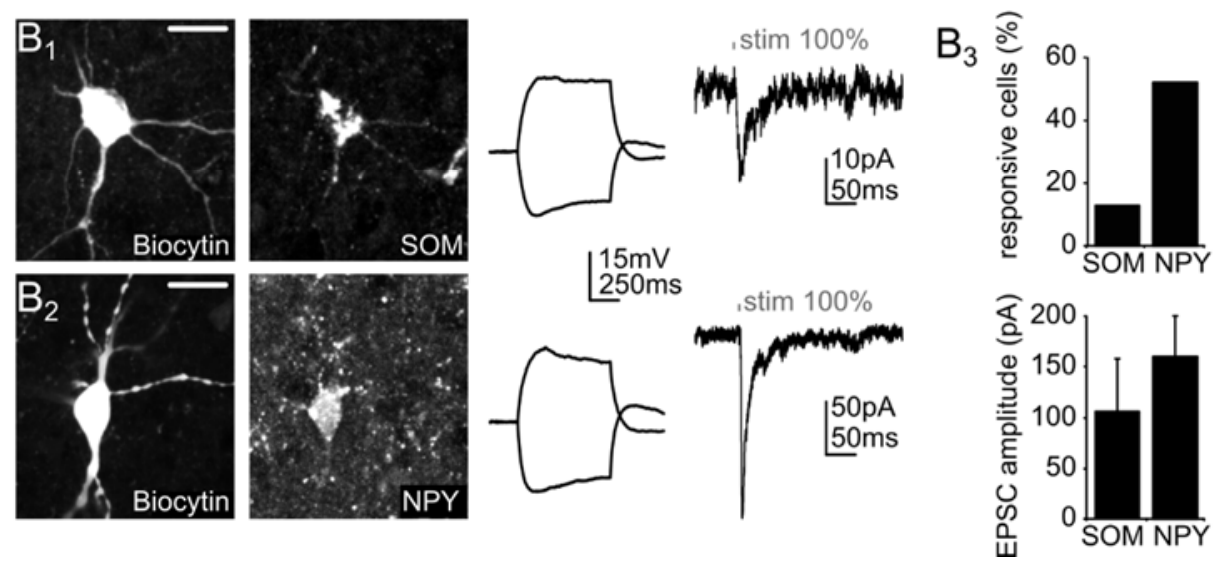


\section{Figure 6}

A

FS Interneuron

Ad Interneuron
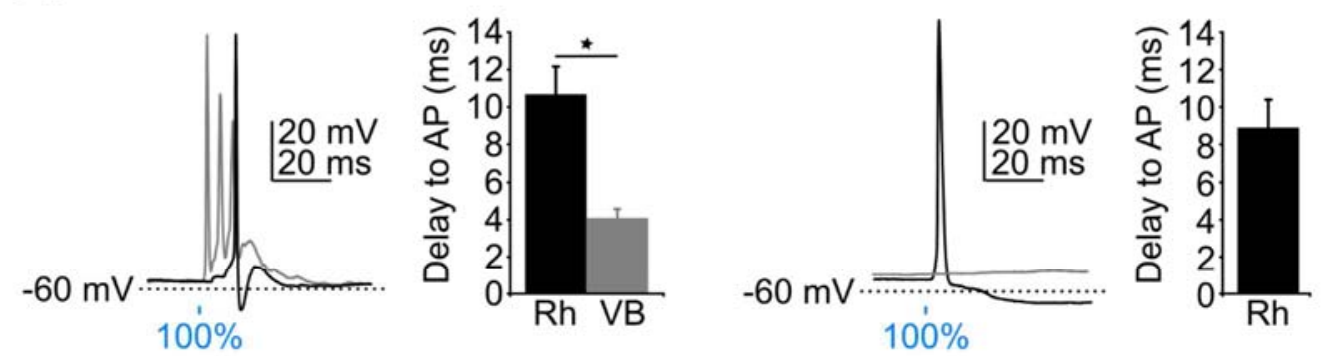

- $\mathrm{Rh}$ (non specific thalamus) VB (specific thalamus)
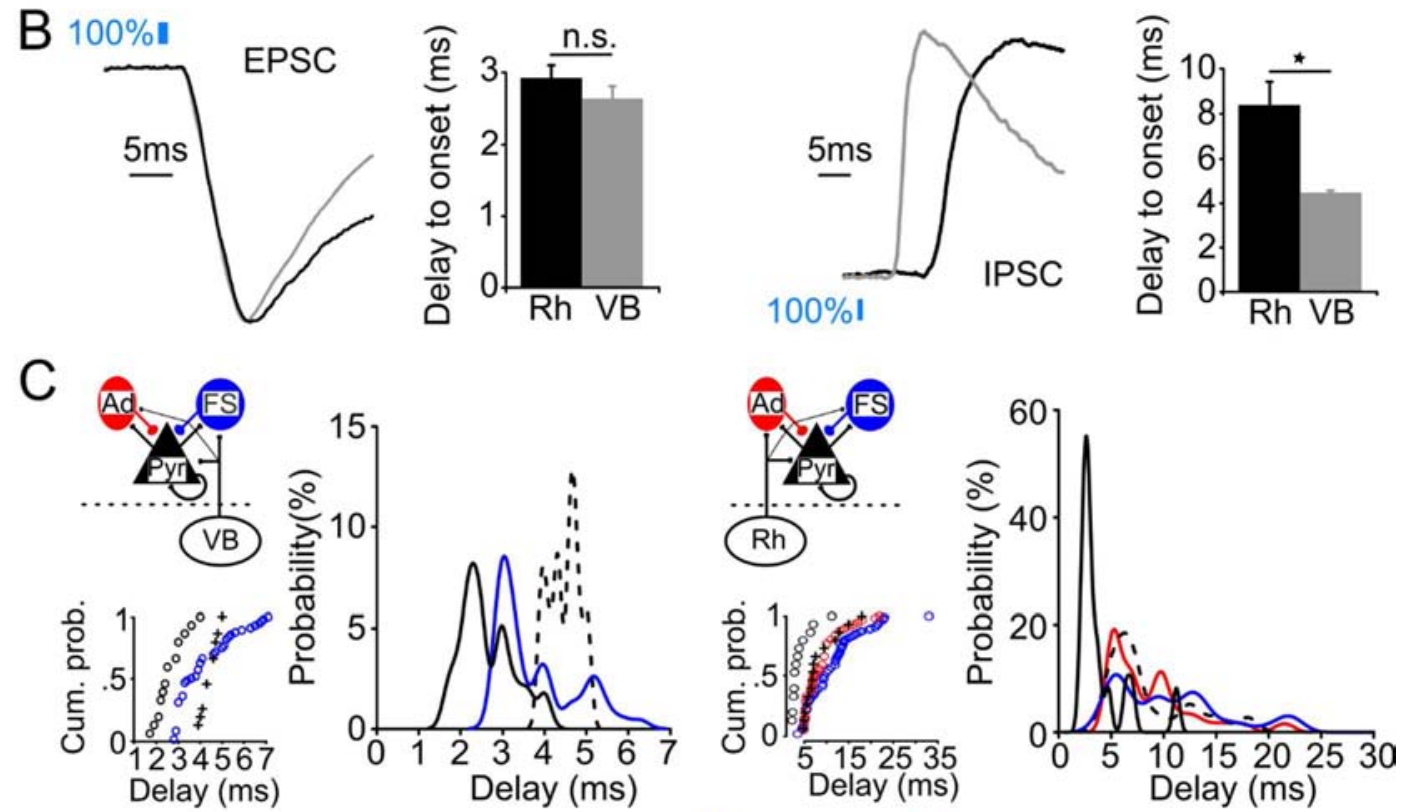

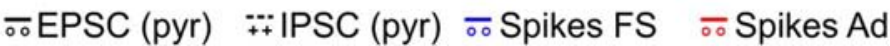




\section{Figure 7}
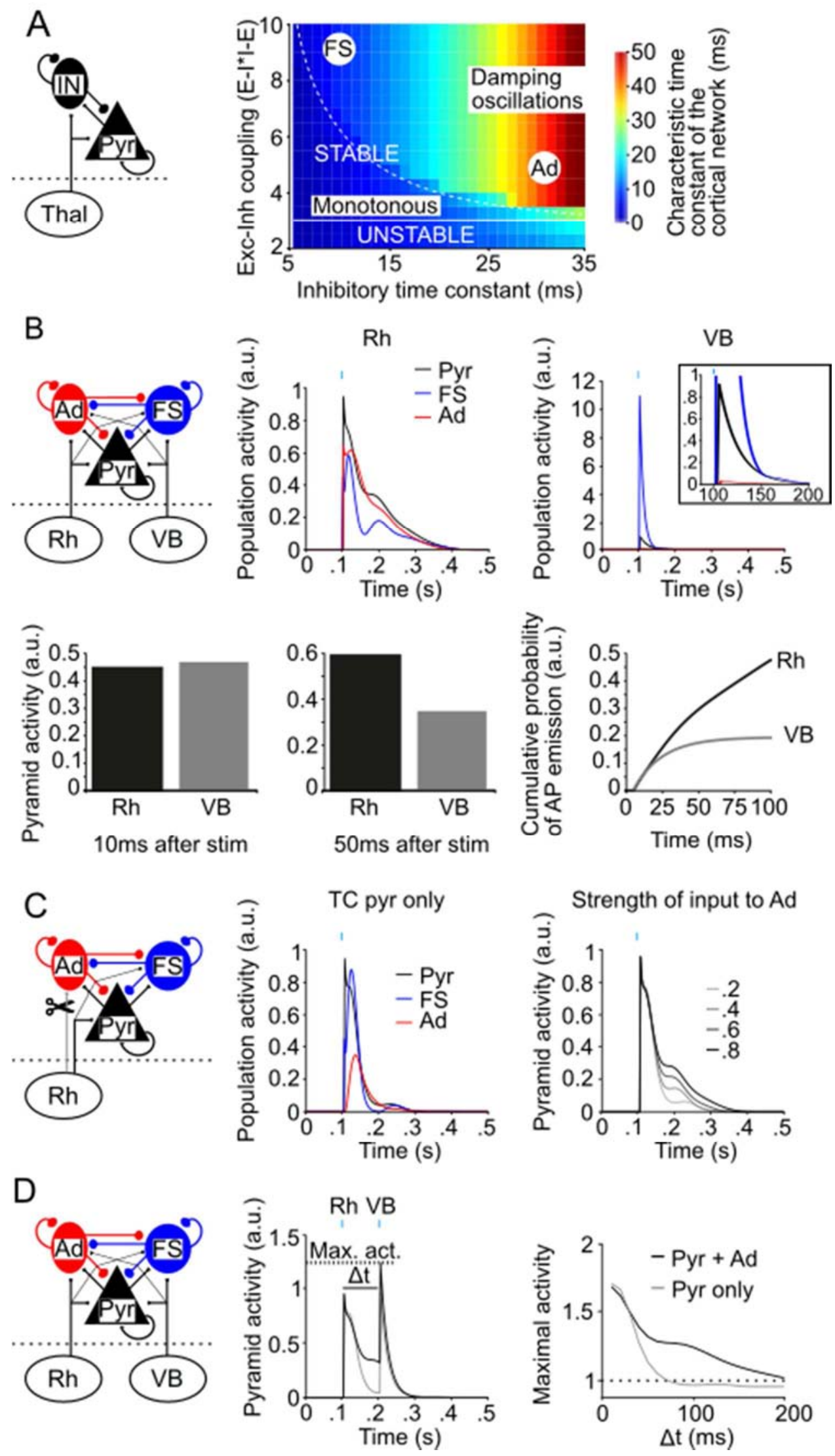


\section{Supplementary Information}

Hay et al. Target Interneuron Preference in Thalamocortical Pathways

$\begin{array}{lc}\text { Supplementary Results__ } & \text { p1 } \\ \text { Supplementary References__ } & \text { p2 } \\ \text { Supplementary Figure Legends } & \text { p3 } \\ \text { Supplementary Figures 1-5 } & \text { pp5-9 }\end{array}$

\section{SUPPLEMENTARY RESULTS}

\section{FS and Ad interneurons exhibit different membrane properties}

Interneurons were identified from their non-pyramidal morphology with aspiny/sparsely spiny dendrites and were classified as parvalbumin-positive or -negative after post-hoc labeling (Suppl. Fig. 3). Parvalbumin-positive interneurons presumably corresponded to FS neurons and conversely, parvalbumin-negative interneurons were considered as Ad interneurons (Kawaguchi 1995, Cauli et al. 1997). Furthermore, electrophysiological properties assessed identification of recorded cells as FS or Ad interneurons. In control conditions with Kgluconate intracellular solution, spike waveforms and firing patterns readily allowed discriminating between the two interneuron classes (Kawaguchi 1995, Cauli et al. 1997), as shown in Suppl. Fig. 3 for their action potential durations $(0.68 \pm 0.03$ vs. $1.36 \pm 0.06 \mathrm{~ms}$ at half amplitude, $\mathrm{p}<0.05)$ and maximal firing rates $(102 \pm 5 \mathrm{vs} .38 \pm 4 \mathrm{~Hz}, \mathrm{p}<0.05$, for FS and Ad interneurons, $\mathrm{n}=13$ and 18, respectively). Notably also, FS interneurons had a lower input resistance than Ad interneurons (223 \pm 31 vs. $361 \pm 26 \mathrm{M} \Omega, \mathrm{n}=13$ and 18, respectively; $\mathrm{p}<$ $0.05)$ and this difference was also observed with Cs-gluconate intracellular solution $(150 \pm 17$ vs. $248 \pm 18 \mathrm{M} \Omega, \mathrm{n}=6$ and 6 , respectively; $\mathrm{p}<0.05)$ or in the presence of TTX-4AP $(198 \pm 17$ vs. $290 \pm 30 \mathrm{M} \Omega, \mathrm{n}=10$ and 10 , respectively; $\mathrm{p}<0.05$ ). Finally, the occurrence of spontaneous EPSCs was higher in FS than in Ad interneurons $(9.4 \pm 1.5$ vs. $2.1 \pm 0.3 \mathrm{~Hz}$, $\mathrm{n}=19$ and 24 , respectively; $\mathrm{p}<0.05)$. 


\section{SUPPLEMENTARY REFERENCES}

Cauli B, Audinat E, Lambolez B, Angulo MC, Ropert N et al. (1997) Molecular and physiological diversity of cortical nonpyramidal cells. J Neurosci. 17:3894-3906

Kawaguchi Y (1995) Physiological subgroups of nonpyramidal cells with specific morphological characteristics in layer II/III of rat frontal cortex. J Neurosci. 15:2638-2655 


\section{SUPPLEMENTARY FIGURE LEGENDS}

\section{Supplementary figure 1: Expression of $\mathrm{ChR2}$ in Rh thalamic neurons}

(A) Whole-field fluorescence negative pictures of serial brain sections along the anteroposterior axis (AP, relative to Bregma) showing expression of ChR2-YFP (black) in the Rh nucleus 4 weeks after viral transduction in a P12 rat. The viral injection site is between AP -2.8 and AP -3.3 sections. The superimposed sections of the rat brain atlas delineates thalamic nuclei (CM: centromedian, Re: reuniens, PVP: paraventricular nucleus posterior part, Sub: submedius). Note that the intense black stripe adjacent to the YFP-labeled Rh nucleus on picture AP -3.8 $\mathrm{mm}$ is an artifact due to damage in this section. (B) Combined infrared and fluorescence picture showing another example of ChR2-YFP expression (green) in the Rh nucleus after viral transduction. (C) Confocal reconstruction (left: $\mathrm{Z}$-stack, right: single plane) of a recorded Rh neuron (red). Note that ChR2-EYFP is present at the plasma membrane of the recorded neuron. (D) Responses of a ChR2-expressing $\mathrm{Rh}$ neuron to depolarizing and hyperpolarizing current steps (left) and to photostimulation (1 ms pulses, blue bars, middle and right panels). (E) Application of orexin $\mathrm{B}$ depolarizes ChR2-expressing Rh neurons and converts responses to photostimulation into a tonic discharge of action potentials.

\section{Supplementary figure 2: Morphology of recorded neurons}

(A) Examples of confocal reconstruction (z-stack) of biocytin-filled excitatory or inhibitory neurons (red) recorded in indicated cortical layers and of ChR2-YFP labeled fibers (green). Note that layer 1 is devoid of excitatory neurons. (B) Examples of confocal reconstruction and immunostaining of biocytin-filled FS and Ad interneurons recorded in layer 6 (PV: parvalbumin, NPY: neuropeptide Y, SOM: somatostatin) and bar graphs summarizing somatodendritic morphologies observed in L6 SOM+ and NPY+ Ad interneurons. SOM+ Ad interneurons exhibited bipolar/bitufted (upper middle panel) or multipolar (upper right panel) morphologies with thick primary dendrites that rarely branched. A majority of NPY+ Ad interneurons were also bipolar/bitufted or multipolar (lower right panel), but some exhibited neurogliaform-like morphology with thin and ramified dendrites (lower middle panel).

\section{Supplementary figure 3: Electrophysiological properties of FS and Ad interneurons}


Upper panels: Biocytin (Bio) labeling of recorded FS and Ad interneurons. The FS interneuron is PV+ and the Ad interneuron is PV-. Traces show spontaneous EPSCs recorded at a holding potential of $-70 \mathrm{mV}$ in these interneurons. The bar graphs summarize EPSC frequency recorded using K-gluconate or Cs-gluconate intracellular solution in FS neurons ( $n=13$ and $n=6$, respectively) or Ad neurons ( $n=18$ and $n=6$, respectively). Upper middle panel: Firing properties of FS $(n=13)$ and $\mathrm{Ad}(\mathrm{n}=18)$ neurons in control conditions. Lower panels: Responses to current injection recorded using K-gluconate intracellular solution in the presence of TTX $(1 \mu \mathrm{M})$ and 4AP $(1 \mathrm{mM})$ in FS $(n=10)$ and Ad $(n=10)$ neurons or using Csgluconate intracellular solution in control conditions in FS $(n=6)$ and $\mathrm{Ad}(n=6)$ interneurons.

\section{Supplementary figure 4: Onset slopes of EPSCs and action potentials in FS and Ad interneurons}

(A) The 20-80\% rise time of short-latency (direct) EPSCs recorded upon $\mathrm{Rh}$ TC stimulation in control conditions is twice slower in $\mathrm{Ad}(73 \pm 34 \mathrm{pA} / \mathrm{ms}, \mathrm{n}=9)$ than in FS interneurons (168 $\pm 64 \mathrm{pA} / \mathrm{ms}, \mathrm{n}=19, \mathrm{p}<0.05$ ). (B) Action potential rise time following $\mathrm{Rh} \mathrm{TC}$ stimulation is slower in Ad $(0.88 \pm 0.04 \mathrm{~ms}, \mathrm{n}=18)$ than in FS interneurons $(0.56 \pm 0.03 \mathrm{~ms}, \mathrm{n}=14, \mathrm{p}<0.05)$. Rise time is measured from the initiation to the peak of the action potential (black bars).

\section{Supplementary figure 5: Differential activation of FS and Ad interneurons leads to contrasting cortical network dynamics in a mean-field model}

(A) Oscillation frequency in the reduced model of excitatory-inhibitory interactions. Null frequencies (black) correspond to non-oscillatory behaviors. (B) Vector fields depicting phase plane portraits of the activity of pyramidal cells and FS or Ad interneurons in the detailed model. Initial strength of VB (mainly activating pyramidal and FS cells, top) and Rh (mainly activating pyramidal and Ad cells, bottom) thalamic inputs on neuronal populations are given by the locations of the arrows. Size of the arrows indicates the speed of the return to initial state. (C) Responses of the pyramidal cell population as a function of $\mathrm{Rh}$ input strength on pyramidal and Ad cells in the detailed model. 


\section{Supplementary Figure 1}

A

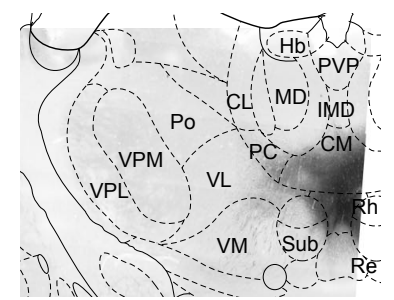

$$
A P=-2.80 \mathrm{~mm}
$$

B

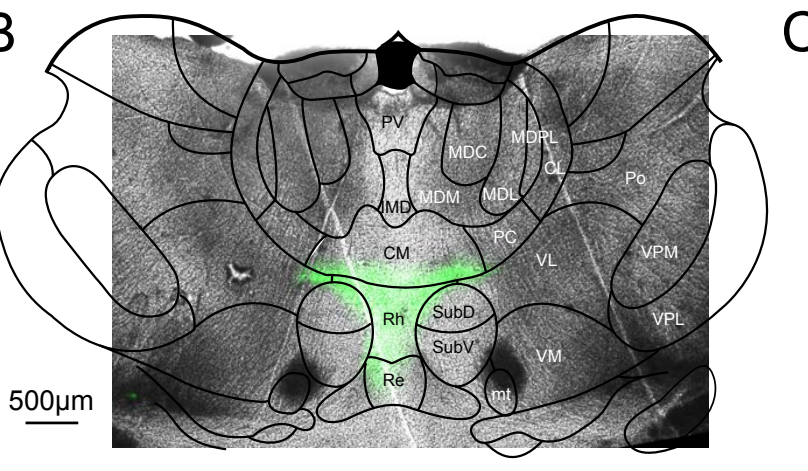

D

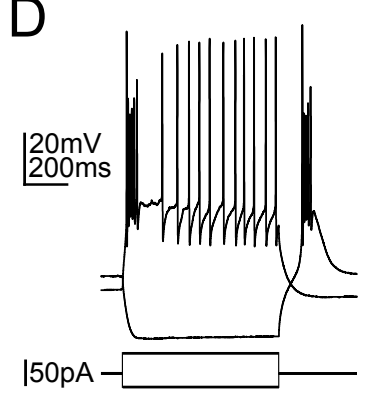

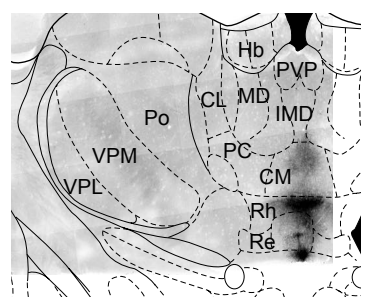

$A P=-3.30 \mathrm{~mm}$

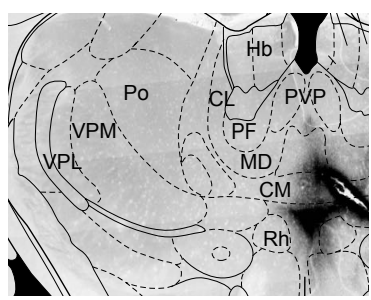

$A P=-3.80 \mathrm{~mm}$

C
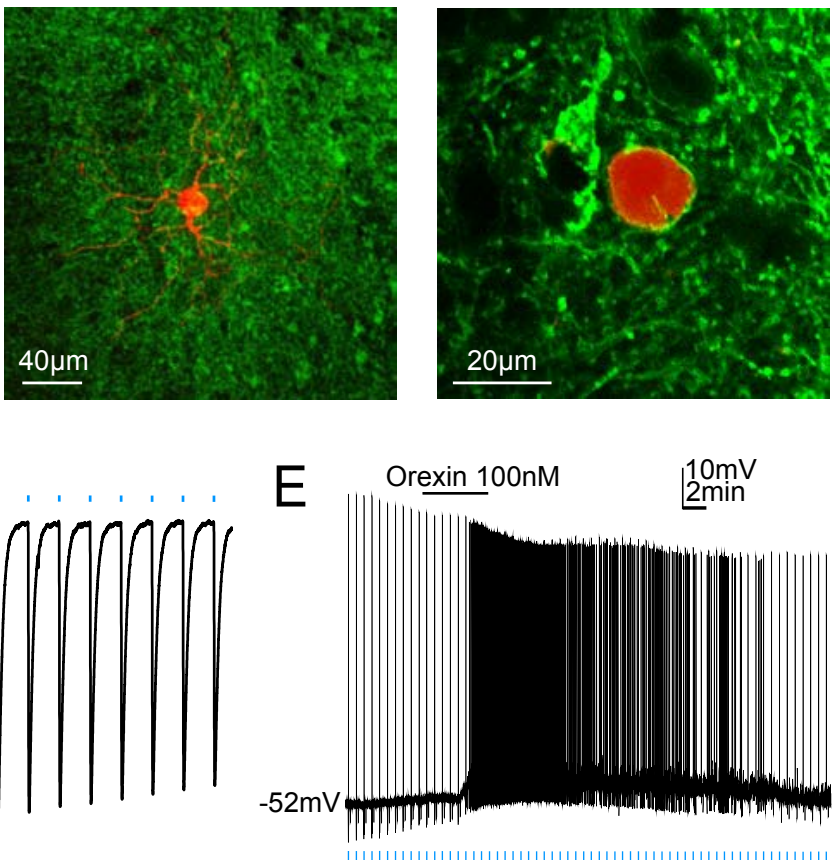


\section{Supplementary Figure 2}

A

Layer 1 IN

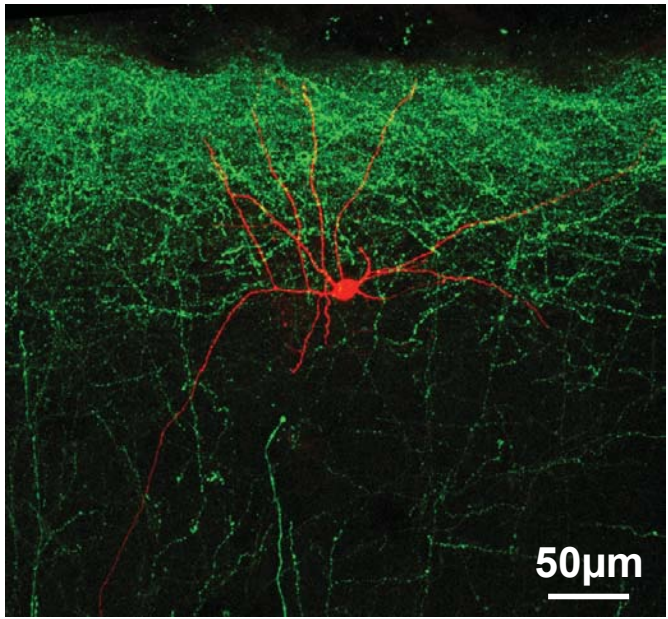

Layer 2/3 Ad IN
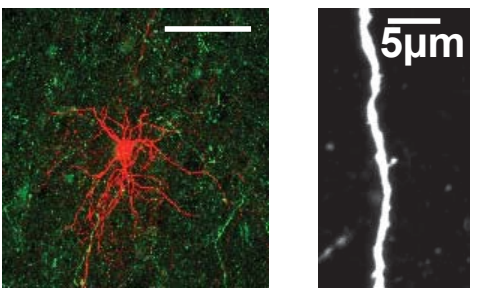

Layer 2 pyramid
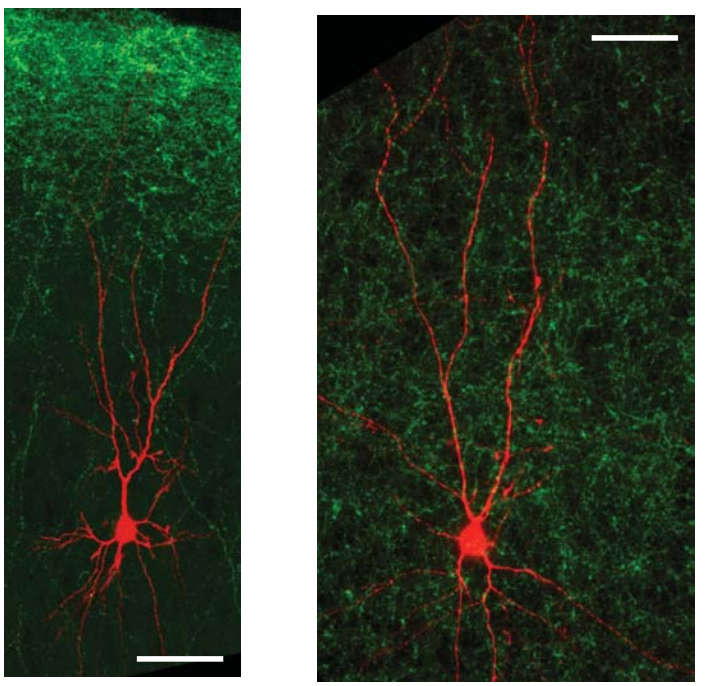

Layer 4
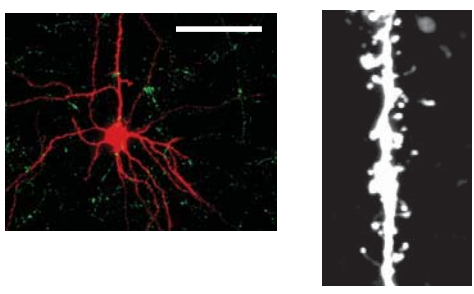

Spiny stellate

Layer 6 pyramid

Layer 6 pyramid

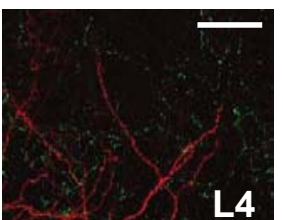

L4

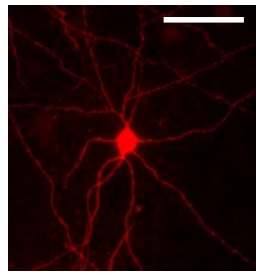

B

Layer 6 FS IN

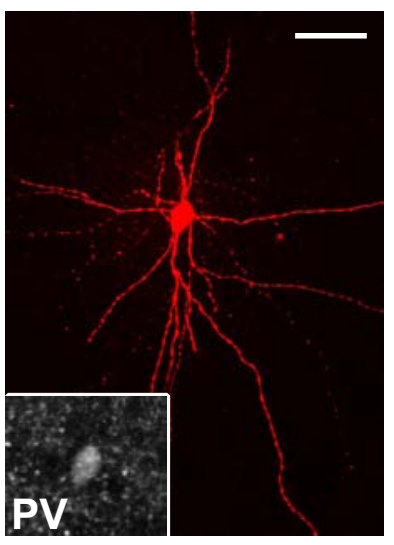

Layer 6 Ad IN
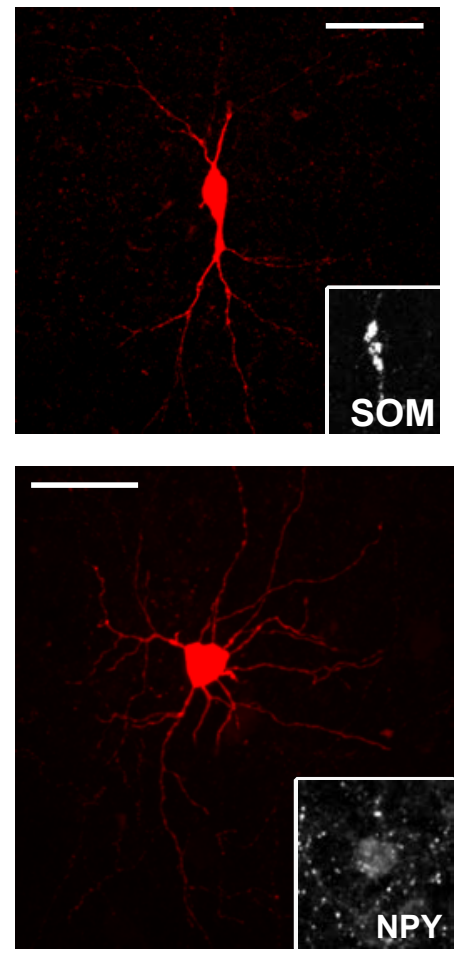
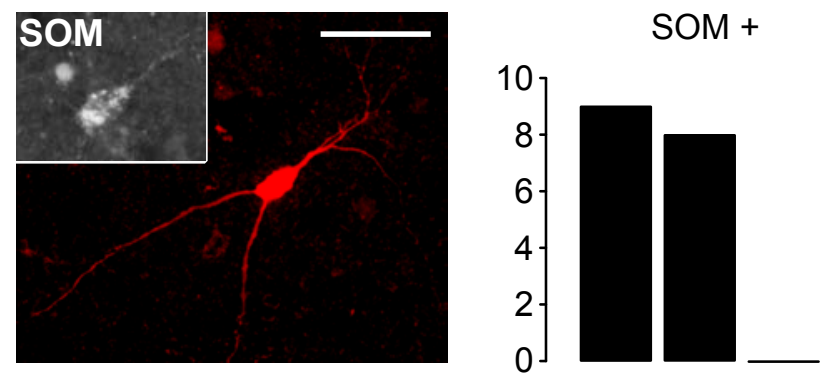

NPY +
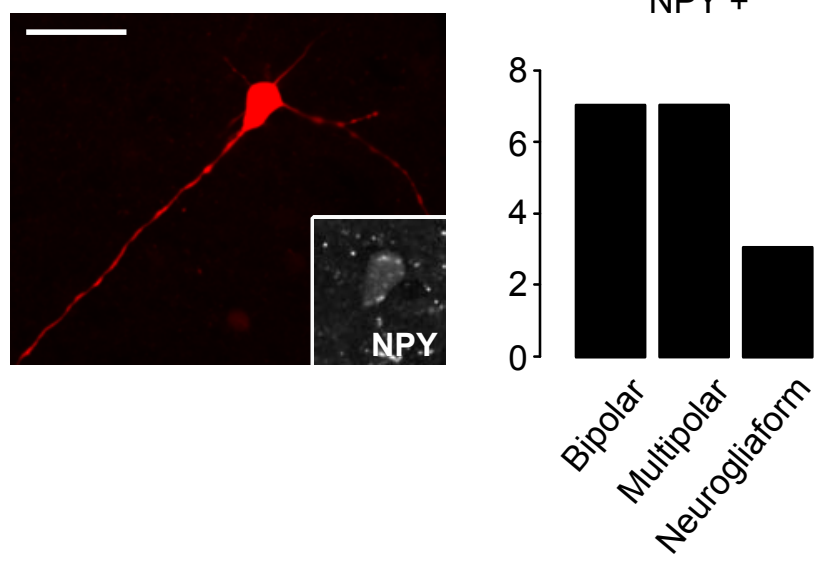


\section{Supplementary Figure 3}

FS
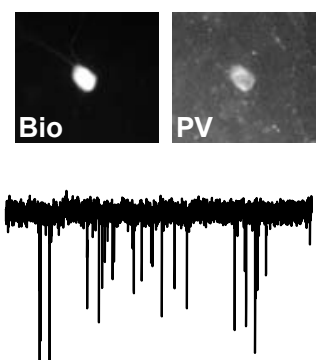

$20 \mathrm{pA}$
$1 \mathrm{~s}$
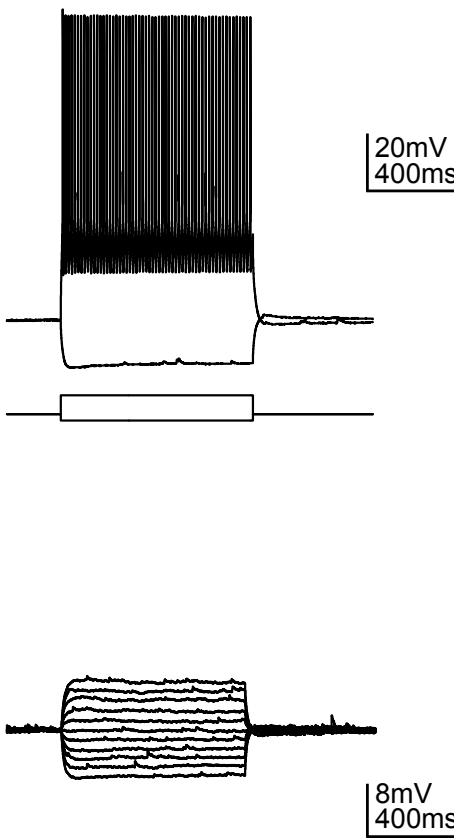

$20 \mathrm{mV}$ 400ms 400ms
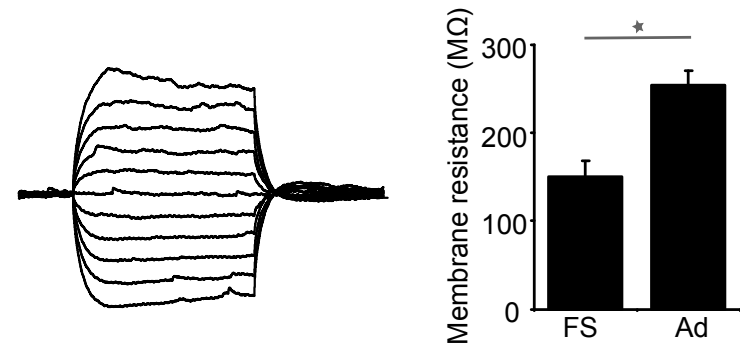

Cesium-based intracellular solution
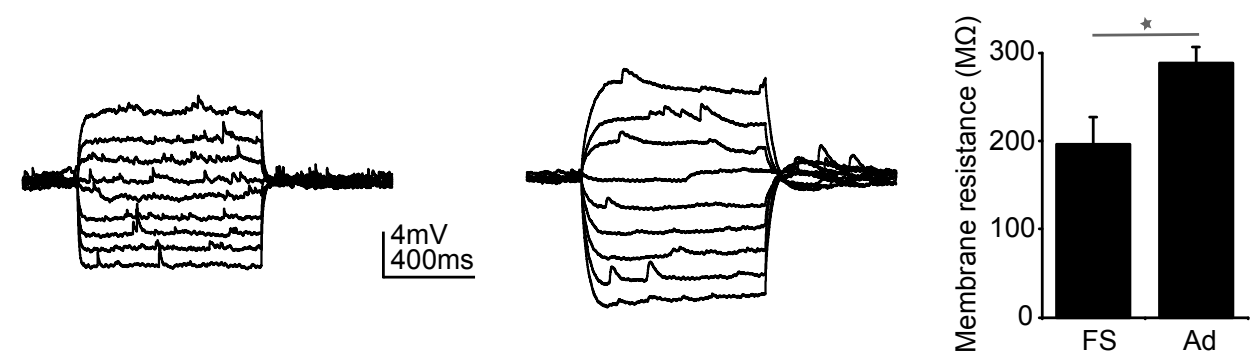

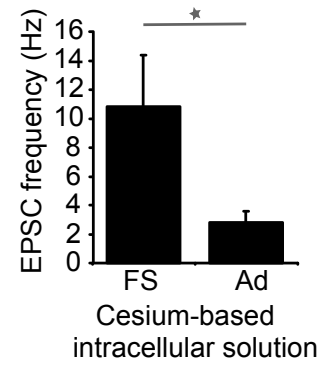

Control conditions
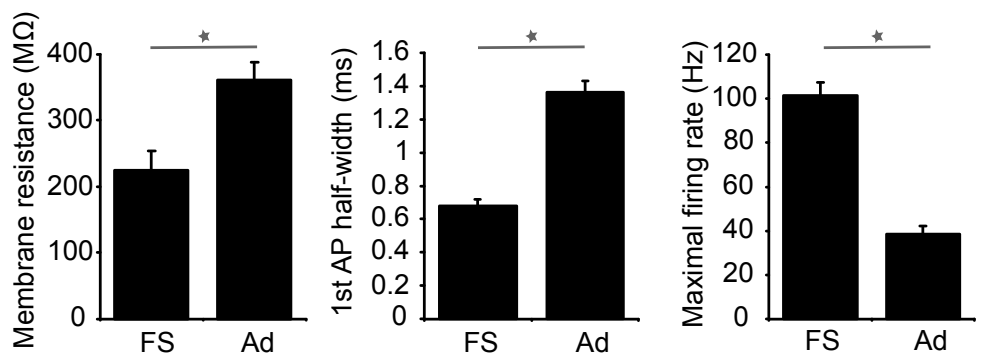


\section{Supplementary Figure 5}

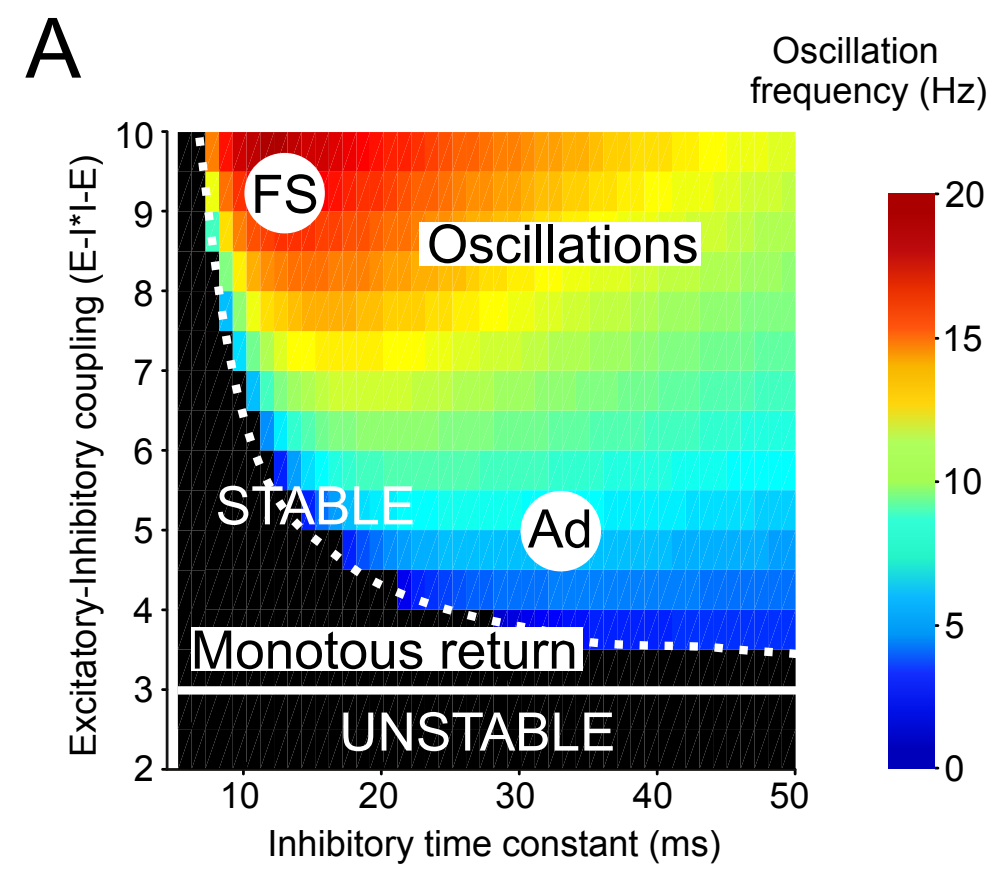

B
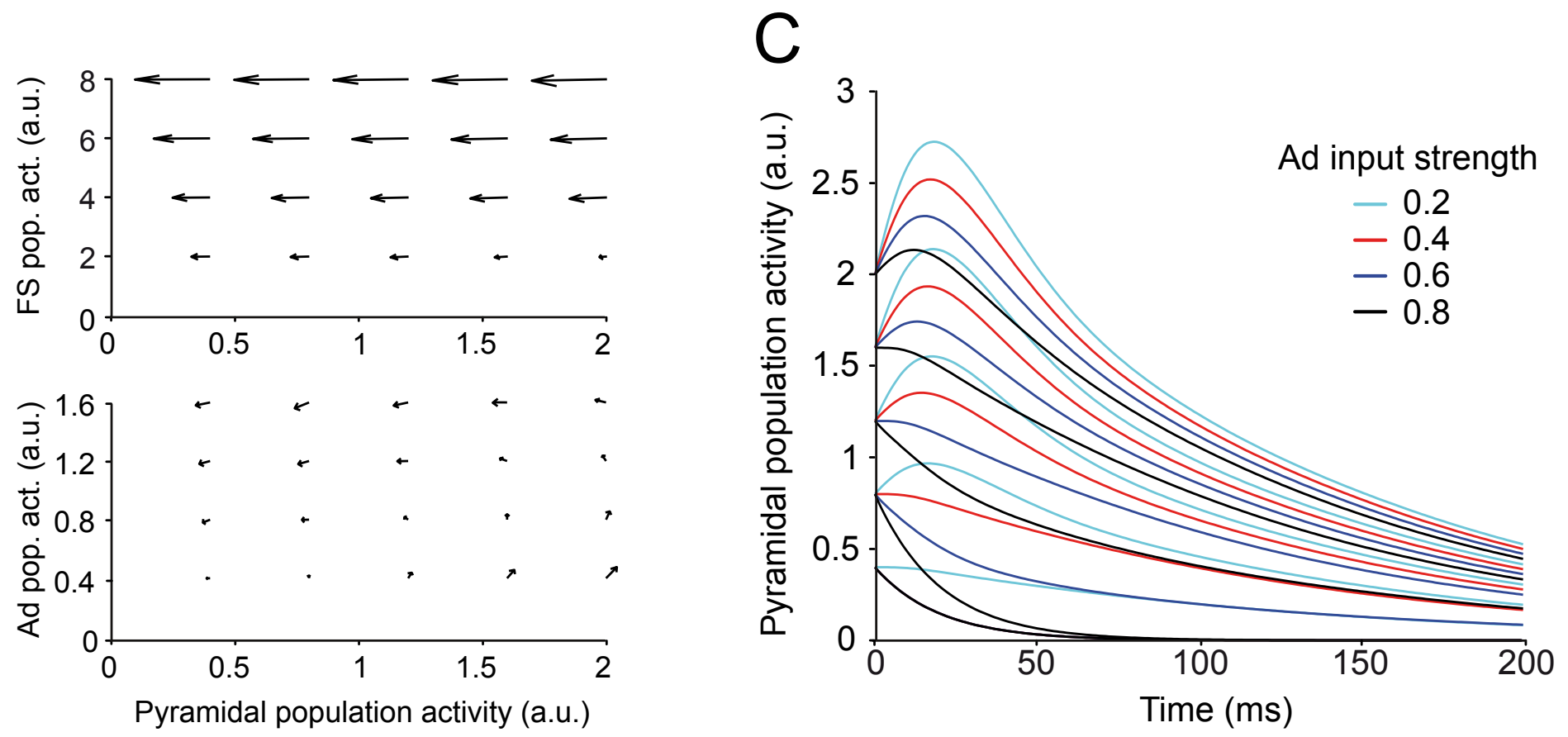